\title{
The Dark Energy Survey: Data Release 1
}

T. M. C. Abbott ${ }^{1}$, F. B. Abdalla ${ }^{2,3}$, S. Allam ${ }^{4}$, A. Amara ${ }^{5}$, J. Annis ${ }^{4}$ (1) J. Asorey ${ }^{6,7,8}$, S. Avila $^{9,10}$, O. Ballester ${ }^{11}$, M. Banerji ${ }^{12,13}$, W. Barkhouse ${ }^{14}$, L. Baruah ${ }^{15}$, M. Baumer ${ }^{16,17,18}$, K. Bechtol ${ }^{19}$, M. R. Becker ${ }^{16,17}$, A. Benoit-Lévy ${ }^{2,20,21}$, G. M. Bernstein ${ }^{22}$, E. Bertin ${ }^{20,21}$, J. Blazek ${ }^{23,24}$, S. Bocquet ${ }^{25}$ (1), D. Brooks ${ }^{(10}$, D. Brout ${ }^{22}$, E. Buckley-Geer ${ }^{4}$, D. L. Burke ${ }^{17,18}$ (1), V. Busti ${ }^{26,27}$, R. Campisano ${ }^{26}$, L. Cardiel-Sas ${ }^{11}$, A. Carnero Rosell ${ }^{26,28}$, M. Carrasco Kind ${ }^{29,30}{ }^{(1)}$, J. Carretero ${ }^{11}$, F. J. Castander ${ }^{31,32}$ (1),

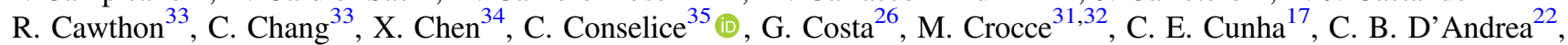
L. N. da Costa $^{26,28}$, R. Das $^{34}$, G. Daues ${ }^{29}$, T. M. Davis ${ }^{7,8}$, C. Davis ${ }^{17}$, J. De Vicente ${ }^{36}$, D. L. DePoy ${ }^{37}$, J. DeRose ${ }^{16,17}$ (10, S. Desai ${ }^{38}$, H. T. Diehl ${ }^{4}$, J. P. Dietrich ${ }^{39,40}$, S. Dodelson ${ }^{41}$, P. Doel ${ }^{2}$, A. Drlica-Wagner ${ }^{4}$ (i), T. F. Eifler ${ }^{42,43}$, A. E. Elliott ${ }^{44}$, A. E. Evrard ${ }^{34,45}$, A. Farahi ${ }^{34}$, A. Fausti Neto ${ }^{26}$, E. Fernandez ${ }^{11}$, D. A. Finley ${ }^{4}$, B. Flaugher ${ }^{4}$, R. J. Foley ${ }^{46}$, P. Fosalba ${ }^{31,32}$, D. N. Friedel ${ }^{29}$, J. Frieman $^{4,33}$, J. García-Bellido ${ }^{10}$ (i), E. Gaztanaga ${ }^{31,32}$, D. W. Gerdes ${ }^{34,45}$ (i), T. Giannantonio ${ }^{12,13,47}$, M. S. S. Gill ${ }^{16,17,18}$, K. Glazebrook ${ }^{6}$ (i), D. A. Goldstein ${ }^{48,49}$ (1), M. Gower ${ }^{29}$, D. Gruen ${ }^{17,18}$ (1), R. A. Gruendl ${ }^{29,30}$ (D) J. Gschwend ${ }^{26,28}$, R. R. Gupta ${ }^{25,48}$,

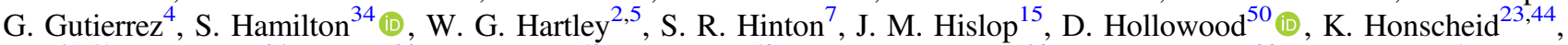
B. Hoyle ${ }^{47,51}$, D. Huterer ${ }^{34}$, B. Jain ${ }^{22}$, D. J. James ${ }^{52}$, T. Jeltema ${ }^{50}$, M. W. G. Johnson ${ }^{29}$, M. D. Johnson ${ }^{29}$, T. Kacprzak ${ }^{5}$, S. Kent ${ }^{4,33}$, G. Khullar ${ }^{33}$, M. Klein ${ }^{40,51}$, A. Kovacs ${ }^{11}$, A. M. G. Koziol' ${ }^{29}$, E. Krause ${ }^{42,43}$, A. Kremin ${ }^{34}$, R. Kron ${ }^{4,53}$, K. Kuehn ${ }^{54}$ (1), S. Kuhlmann ${ }^{25}$, N. Kuropatkin ${ }^{4}$, O. Lahav ${ }^{2}$, J. Lasker ${ }^{33,53}$, T. S. Li ${ }^{4}$, R. T. Li ${ }^{29}$, A. R. Liddle ${ }^{55}$, M. Lima ${ }^{26,27}$, H. Lin ${ }^{4}$, P. López-Reyes ${ }^{36}$, N. MacCrann ${ }^{23,44}$, M. A. G. Maia ${ }^{26,28}$, J. D. Maloney ${ }^{29}$, M. Manera ${ }^{11,74}$, M. March ${ }^{22}$, J. Marriner ${ }^{4}$,

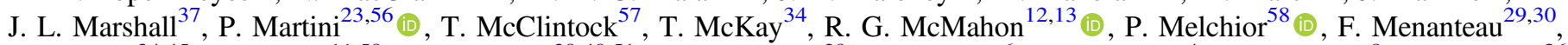
C. J. Miller ${ }^{34,45}$, R. Miquel ${ }^{11,59}$ (1) J. J. Mohr ${ }^{39,40,51}$, E. Morganson ${ }^{29}$ (i), J. Mould ${ }^{6}$ (i) , E. Neilsen ${ }^{4}$, R. C. Nichol ${ }^{9}$, F. Nogueira ${ }^{26}$,

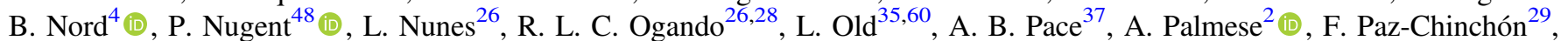

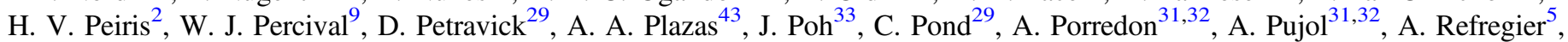
K. Reil ${ }^{18}$, P. M. Ricker ${ }^{29,30}$ (1) , R. P. Rollins ${ }^{61}$, A. K. Romer $^{15}$ (i), A. Roodman ${ }^{17,18}$ (1) , P. Rooney ${ }^{15}$, A. J. Ross ${ }^{23}$, E. S. Rykoff ${ }^{17,18}$, M. Sako ${ }^{22}$, M. L. Sanchez ${ }^{26}$, E. Sanchez ${ }^{36}$, B. Santiago ${ }^{26,62}$, A. Saro ${ }^{40,63}$, V. Scarpine ${ }^{4}$, D. Scolnic ${ }^{33}$, S. Serrano ${ }^{31,32}$, I. Sevilla-Noarbe ${ }^{36}$, E. Sheldon ${ }^{64}$, N. Shipp ${ }^{33}$ (1) M. L. Silveira ${ }^{26}$, M. Smith ${ }^{65}$ (D), R. C. Smith ${ }^{1}$, J. A. Smith ${ }^{66}$,

M. Soares-Santos ${ }^{4,67}$ (i), F. Sobreira ${ }^{26,68}$ () , J. Song ${ }^{69}$, A. Stebbins ${ }^{4}$, E. Suchyta ${ }^{70}$, M. Sullivan ${ }^{65}$ (1), M. E. C. Swanson ${ }^{29}$, G. Tarle $^{34}$ (10) J. Thaler ${ }^{71}$, D. Thomas ${ }^{9}$, R. C. Thomas ${ }^{48}$, M. A. Troxel ${ }^{23,44}$, D. L. Tucker ${ }^{4}$, V. Vikram ${ }^{25}$, A. K. Vivas ${ }^{1}$ (1),

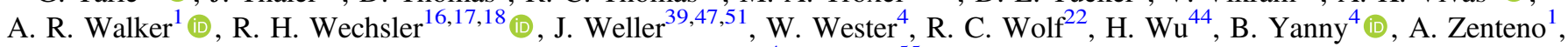
Y. Zhang ${ }^{4}$, J. Zuntz ${ }^{55}$

(DES Collaboration),

and

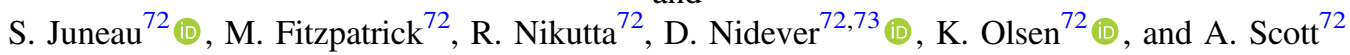
(NOAO Data Lab)

\footnotetext{
${ }^{1}$ Cerro Tololo Inter-American Observatory, National Optical Astronomy Observatory, Casilla 603, La Serena, Chile

${ }^{2}$ Department of Physics \& Astronomy, University College London, Gower Street, London, WC1E 6BT, UK

${ }^{3}$ Department of Physics and Electronics, Rhodes University, P.O. Box 94, Grahamstown, 6140, South Africa ${ }^{4}$ Fermi National Accelerator Laboratory, P.O. Box 500, Batavia, IL 60510, USA

${ }^{5}$ Department of Physics, ETH Zurich, Wolfgang-Pauli-Strasse 16, CH-8093 Zurich, Switzerland

${ }^{6}$ Centre for Astrophysics \& Supercomputing, Swinburne University of Technology, Victoria 3122, Australia ${ }^{7}$ School of Mathematics and Physics, University of Queensland, Brisbane, QLD 4072, Australia ${ }^{8}$ ARC Centre of Excellence for All-sky Astrophysics (CAASTRO)

${ }^{9}$ Institute of Cosmology \& Gravitation, University of Portsmouth, Portsmouth, PO1 3FX, UK

${ }^{10}$ Instituto de Fisica Teorica UAM/CSIC, Universidad Autonoma de Madrid, E-28049 Madrid, Spain

${ }^{11}$ Institut de Física d'Altes Energies (IFAE), The Barcelona Institute of Science and Technology, Campus UAB, E-08193 Bellaterra (Barcelona) Spain

${ }^{12}$ Kavli Institute for Cosmology, University of Cambridge, Madingley Road, Cambridge CB3 OHA, UK

${ }^{13}$ Institute of Astronomy, University of Cambridge, Madingley Road, Cambridge CB3 OHA, UK

${ }^{14}$ University of North Dakota, Department of Physics and Astrophysics, Witmer Hall, Grand Forks, ND 58202, USA

${ }^{15}$ Department of Physics and Astronomy, Pevensey Building, University of Sussex, Brighton, BN1 9QH, UK

${ }^{16}$ Department of Physics, Stanford University, 382 Via Pueblo Mall, Stanford, CA 94305, USA

${ }^{17}$ Kavli Institute for Particle Astrophysics \& Cosmology, P.O. Box 2450, Stanford University, Stanford, CA 94305, USA ${ }^{18}$ SLAC National Accelerator Laboratory, Menlo Park, CA 94025, USA

${ }^{19}$ LSST, 933 North Cherry Avenue, Tucson, AZ 85721, USA; kbechtol@1sst.org

${ }^{20}$ Sorbonne Universités, UPMC Univ Paris 06, UMR 7095, Institut d'Astrophysique de Paris, F-75014, Paris, France ${ }^{21}$ CNRS, UMR 7095, Institut d'Astrophysique de Paris, F-75014, Paris, France

${ }^{22}$ Department of Physics and Astronomy, University of Pennsylvania, Philadelphia, PA 19104, USA

${ }^{23}$ Center for Cosmology and Astro-Particle Physics, The Ohio State University, Columbus, OH 43210, USA

${ }^{24}$ Institute of Physics, Laboratory of Astrophysics, École Polytechnique Fédérale de Lausanne (EPFL), Observatoire de Sauverny, 1290 Versoix, Switzerland ${ }^{25}$ Argonne National Laboratory, 9700 South Cass Avenue, Lemont, IL 60439, USA

${ }^{26}$ Laboratório Interinstitucional de e-Astronomia-LIneA, Rua Gal. José Cristino 77, Rio de Janeiro, RJ-20921-400, Brazil

${ }^{27}$ Departamento de Física Matemática, Instituto de Física, Universidade de São Paulo, CP 66318, São Paulo, SP, 05314-970, Brazil ${ }^{28}$ Observatório Nacional, Rua Gal. José Cristino 77, Rio de Janeiro, RJ-20921-400, Brazil

${ }^{29}$ National Center for Supercomputing Applications, 1205 West Clark St., Urbana, IL 61801, USA; mcarras2@illinois.edu

${ }^{30}$ Department of Astronomy, University of Illinois at Urbana-Champaign, 1002 W. Green Street, Urbana, IL 61801, USA
} 


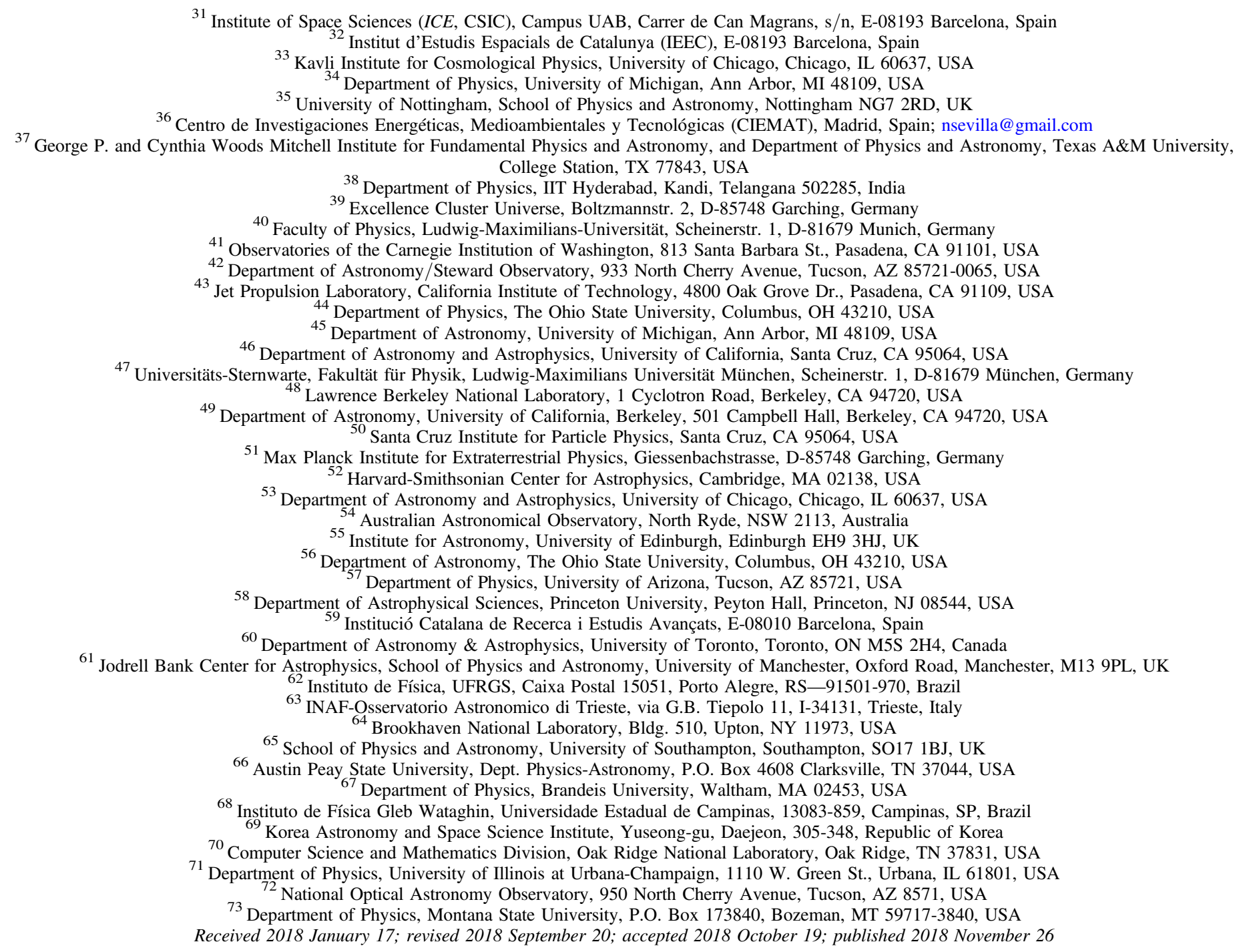

\begin{abstract}
We describe the first public data release of the Dark Energy Survey, DES DR1, consisting of reduced single-epoch images, co-added images, co-added source catalogs, and associated products and services assembled over the first $3 \mathrm{yr}$ of DES science operations. DES DR1 is based on optical/near-infrared imaging from 345 distinct nights (2013 August to 2016 February) by the Dark Energy Camera mounted on the $4 \mathrm{~m}$ Blanco telescope at the Cerro Tololo InterAmerican Observatory in Chile. We release data from the DES wide-area survey covering $\sim 5000 \mathrm{deg}^{2}$ of the southern Galactic cap in five broad photometric bands, griz Y. DES DR1 has a median delivered point-spread function of $g=1.12, r=0.96, i=0.88, z=0.84$, and $Y=0$ " $90 \mathrm{FWHM}$, a photometric precision of $<1 \%$ in all bands, and an astrometric precision of 151 mas. The median co-added catalog depth for a 1."95 diameter aperture at signal-to-noise ratio $(\mathrm{S} / \mathrm{N})=10$ is $g=24.33, r=24.08, i=23.44, z=22.69$, and $Y=21.44 \mathrm{mag}$. DES DR1 includes nearly 400 million distinct astronomical objects detected in $\sim 10,000$ co-add tiles of size $0.534 \mathrm{deg}^{2}$ produced from $\sim 39,000$ individual exposures. Benchmark galaxy and stellar samples contain $\sim 310$ million and $\sim 80$ million objects, respectively, following a basic object quality selection. These data are accessible through a range of interfaces, including query web clients, image cutout servers, jupyter notebooks, and an interactive co-add image visualization tool. DES DR1 constitutes the largest photometric data set to date at the achieved depth and photometric precision.
\end{abstract}

Key words: astronomical databases: miscellaneous - catalogs - cosmology: observations - surveys - techniques: image processing - techniques: photometric

\title{
1. Introduction
}

$\overline{74}$ Visitor at Kavli Institute for Cosmology, University of Cambridge, Madingley Road, Cambridge CB3 0HA.
Advances in telescope construction, sensor technology, and data processing have allowed us to map the sky with increasing speed and precision, enabling discovery through statistical 
analysis of astronomical source populations, as well as the detection of rare and/or unexpected objects (Tyson 2010). The Dark Energy Survey (DES) is one of several ground-based wide-area optical and near-IR imaging surveys, including the Sloan Digital Sky Survey (SDSS; York et al. 2000), the Panoramic Survey Telescope and Rapid Response System 1 (Pan-STARRS1 or PS1; Kaiser et al. 2010), the Kilo Degree Survey (KiDS; de Jong et al. 2013), the Hyper Suprime-Cam Subaru Strategic Program (HSC-SSP; Aihara et al. 2018a), and the future Large Synoptic Survey Telescope (LSST; Ivezic et al. 2008).

The instrumental and observational strategies of DES are designed to improve our understanding of cosmic acceleration and the nature of dark energy using four complementary methods: weak gravitational lensing, galaxy cluster counts, the large-scale clustering of galaxies (including baryon acoustic oscillations), and the distances to Type Ia supernovae ( $\mathrm{SNe}$; DES Collaboration 2005). To achieve these goals, DES conducts two distinct multiband imaging surveys: a $\sim 5000 \mathrm{deg}^{2}$ widearea survey in the grizY bands and a $\sim 27 \mathrm{deg}^{2}$ deep SN survey observed in the griz bands with a $\sim 7$-day cadence (Diehl et al. 2014; Kessler et al. 2015).

DES uses the Dark Energy Camera (DECam; Honscheid et al. 2008; Flaugher et al. 2015), a 570 MP camera with a $3 \mathrm{deg}^{2}$ field of view installed at the prime focus of the Blanco 4 $\mathrm{m}$ telescope at the Cerro Tololo Inter-American Observatory (CTIO) in northern Chile. Survey observations comprise $~ 105$ equivalent full nights per year (August through mid-February), including full and half nights. Each exposure is delivered from CTIO to the National Center for Supercomputing Applications (NCSA) at the University of Illinois at Urbana-Champaign for processing generally within minutes of being observed. At NCSA, the DES Data Management system (DESDM; Sevilla et al. 2011; Desai et al. 2012; Mohr et al. 2012; Morganson et al. 2018) generates a variety of scientific products, including single-epoch and co-added images with associated source catalogs of suitable quality to perform precise cosmological measurements (e.g., DES Collaboration 2017).

Raw DES exposures become publicly available $1 \mathrm{yr}$ after acquisition from the National Optical Astronomy Observatory (NOAO) Science Archive, ${ }^{75}$ and DES is scheduled to provide two major public releases of processed data. The first DES Data Release (DR1), described here, encompasses data products derived from wide-area survey observations taken in the first 3 yr of science operations (Y1-Y3, from 2013 August to 2016 February). A second major data release (DR2) is scheduled for after DES is completed. In addition to DR1 and DR2, the DES Collaboration prepares incremental internal releases with value-added products and detailed characterizations of survey performance that are designed to support cosmological analyses (e.g., Y1 Gold; Drlica-Wagner et al. 2018). A subset of these products associated with data collected during the DES Science Verification (SV) period (2012 November 1 through 2013 February 22) was released in 2016 January. ${ }^{76}$ In 2018 September, the value-added products from a number of selected DES publications corresponding to $\mathrm{Y} 1$ data were released as well. ${ }^{77}$ Additional releases of value-added data products are expected to support future scientific publications.

\footnotetext{
75 http://archive.noao.edu/

76 https://des.ncsa.illinois.edu/releases/sva1

77 https://des.ncsa.illinois.edu/releases/y1a1
}

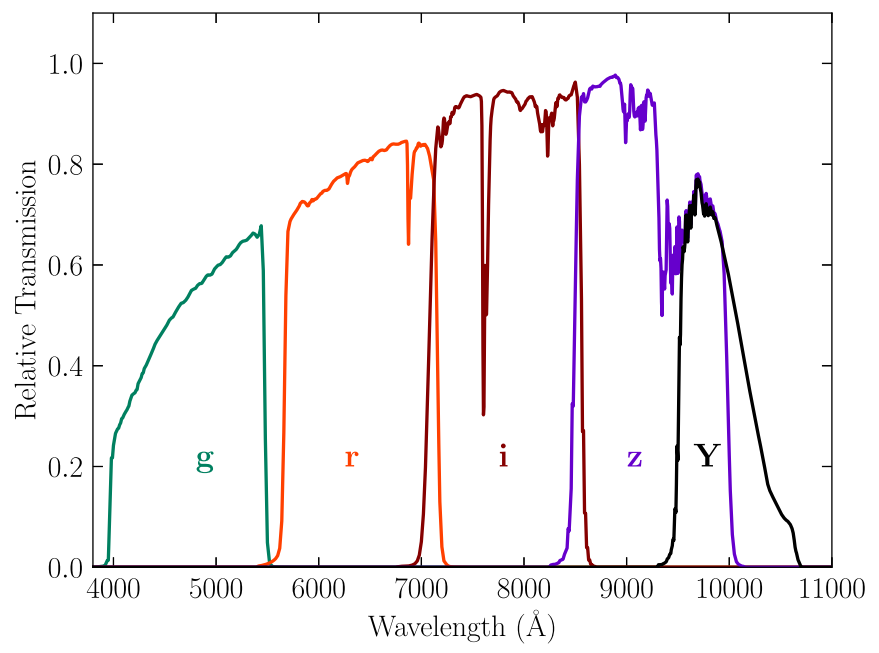

Figure 1. DR1 standard bandpasses for the DECam grizY filters. The bandpasses represent the total system throughput, including atmospheric transmission (air mass $=1.2$ ) and the average instrumental response across the science CCDs (Section 5.4).

In this work, we present the content, validation, and data access services for DES DR1. DR1 is composed of co-added images and catalogs, as well as calibrated single-epoch images, from the processing of the first $3 \mathrm{yr}$ of DES wide-area survey observations. Access to DES DR1 data is provided via web interfaces and auxiliary tools, which is made possible through the partnership between $\mathrm{NCSA}^{78}{ }^{7 \text { LneA, }}{ }^{79}$ and NOAO, ${ }^{80}$ at the following URL: https://des.ncsa.illinois.edu/releases/dr1. In Section 2, we briefly describe the DECam instrument and the DES observation strategy for the wide-field survey (the data set included in this release). Section 3 includes an overview of how the raw data were processed by DESDM at NCSA and served as the catalogs and images made available in this release. A basic quality evaluation of these products is presented in Section 4, followed by a description of products as they appear in the DR1 release (Section 5). Section 6 describes the various data access frameworks and tools made available for DR1. A summary of the release and information on expected future releases are given in Section 7 . We direct the reader to Appendix A for definitions of terms and acronyms used throughout the text.

Except where noted, all magnitudes quoted in the text are in the AB system (Oke 1974).

\section{Data Acquisition}

DR1 is composed of data taken on 345 distinct nights spread over the first $3 \mathrm{yr}$ of DES operations from 2013 August 15 to 2016 February $12 .{ }^{81}$ In this section, we briefly describe the characteristics of the DECam instrument and the DES observation strategy to provide context for DR1. We point the reader to other DES publications for further details on the technical aspects summarized here (i.e., Diehl et al. 2016; Drlica-Wagner et al. 2018; Morganson et al. 2018).

\footnotetext{
78 National Center for Supercomputing Applications.

79 Laboratório Interinstitucional de e-Astronomia.

80 National Optical Astronomy Observatory.

81 DES was scheduled for 319 equivalent full nights, including half nights, during this period (Diehl et al. 2016).
} 


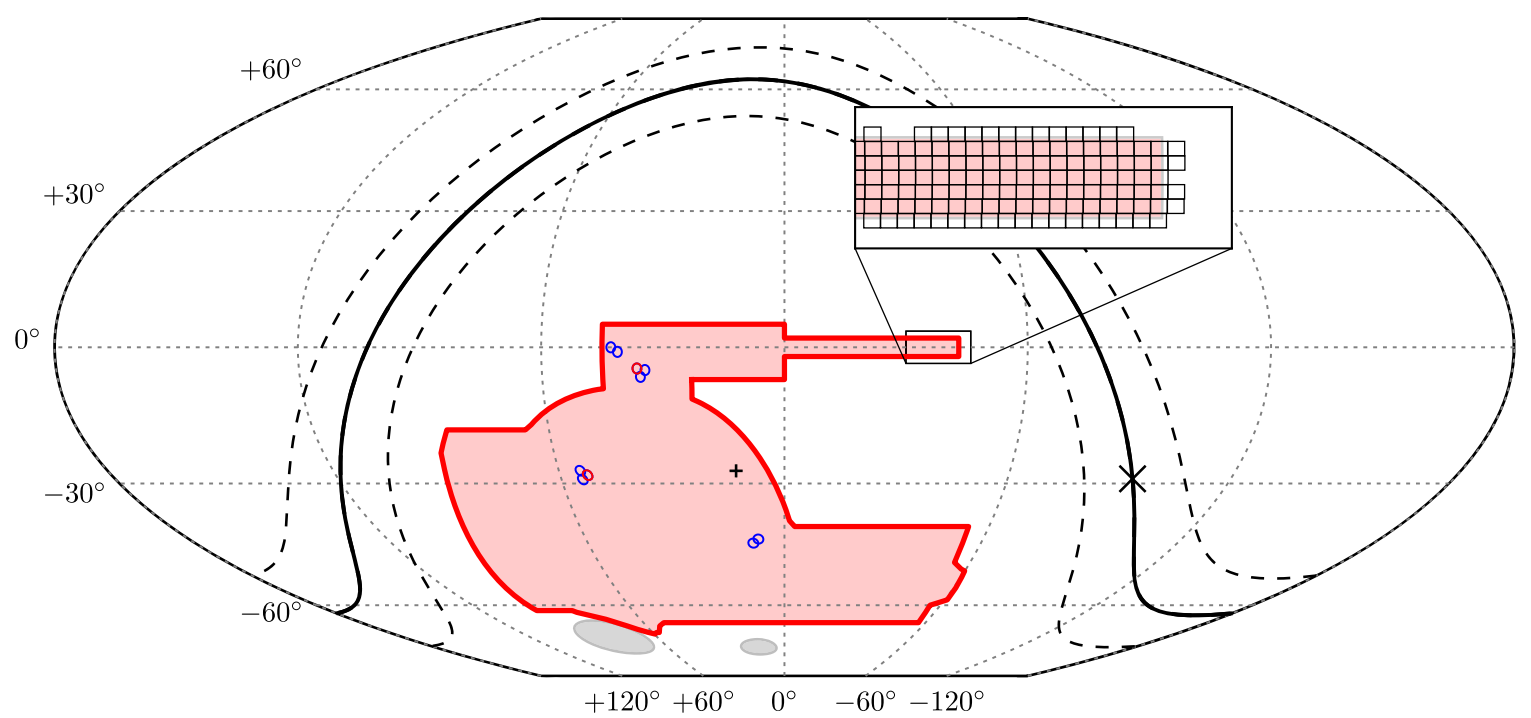

Figure 2. Plot of the DES survey area in celestial coordinates. The $\sim 5000 \mathrm{deg}^{2}$ wide-area survey footprint is shown in red. The eight shallow SN fields are shown as blue circles, and the two deep SN fields are shown as red circles. The Milky Way plane is shown as a solid line, with dashed lines at $b= \pm 10$ deg. The Galactic center (cross) and south Galactic pole (plus sign) are also marked. The Large and Small Magellanic Clouds are indicated in gray. The inset panel shows an overlay of co-add processing units, co-add tiles, on top of the SDSS Stripe 82 area. This and the other sky map plots included in this work use the equal-area McBryde-Thomas flatpolar quartic projection.

\subsection{DECam}

DECam is a wide-field-of-view $\left(3 \mathrm{deg}^{2}\right)$ mosaic camera containing 62 science CCDs (Flaugher et al. 2015). ${ }^{82}$ The corrector system and pixel size provide an average plate scale of 0 ". 263 per pixel. The DES wide-area survey observes five broadband filters, grizY (Figure 1), and the standard bandpasses for these filters are included as part of DR1 (Section 5.4). The DES filters are very similar to their analogously named counterparts from other surveys.

Uniquely, the DES $z$ band has greater sensitivity at longer wavelengths than the SDSS $z$ band and overlaps with the DES $Y$ band. Additional details, including construction, installation, and a description of DECam subsystems and interfaces, are provided in Flaugher et al. (2015).

\subsection{Survey Operations}

The target footprint of the DES wide-area and SN surveys are shown in Figure 2. All R.A., decl. coordinates in this paper refer to the J2000 epoch. The wide-area footprint shape was selected to obtain a large overlap with the South Pole Telescope survey (Carlstrom et al. 2011) and Stripe 82 from SDSS (Abazajian et al. 2009) and includes a connection region to enhance overall calibration. Given the cosmological goals of the survey, DES avoids the Galactic plane to minimize stellar foregrounds and extinction from interstellar dust.

The wide-field survey uses exposure times of $90 \mathrm{~s}$ for griz and $45 \mathrm{~s}$ for $Y$ band, yielding a typical single-epoch point-spread function (PSF) depth at signal-to-noise ratio $(\mathrm{S} / \mathrm{N})=10$ of $g=23.57$, $r=23.34, \quad i=22.78, \quad z=22.10$, and $Y=20.69$ (Morganson et al. 2018). The completed survey is expected to be roughly 1 mag deeper, through the co-addition of 10 images in each of the bands for a cumulative exposure time of $900 \mathrm{~s}$ in griz and $450 \mathrm{~s}$ in $Y^{83}$

\footnotetext{
82 Two and a half DECam CCDs have failed over the course of DES operation and are only included in DR1 when operating properly (Diehl et al. 2014; Flaugher et al. 2015; Morganson et al. 2018).

83 Beginning in $\mathrm{Y} 4, \mathrm{Y}$-band exposure times were increased to $90 \mathrm{~s}$ to reduce overhead while maintaining the same cumulative exposure target.
}

Nightly observations are divided between the wide-field and SN surveys based on current environmental conditions and the data quality assessments of previous observations. Real-time optimization of survey strategy is accomplished through the ObsTac software on the mountain (Neilsen \& Annis 2014). ObsTac selects grizY exposures accounting for moon position, sky brightness, current seeing, air mass, hour angle, and other observational characteristics. DES exposures are offset by roughly half the focal plane radius on average in successive visits to the same field, or "hex," such that objects are observed by different CCDs in each "tiling." This observing strategy minimizes inhomogeneities from the DECam geometry and enhances the relative photometric calibration. Blanco is an equatorial mount telescope, and there is no rotation between dithered and/or repeated exposures.

A single raw DECam exposure is $\sim 0.5 \mathrm{~GB}$ in size (compressed), and DES collects $\sim 300$ science exposures per night, depending on the season, survey strategy, and SN field schedule. These data are transferred to NOAO for archiving (Fitzpatrick 2010; Honscheid et al. 2012) and to NCSA for further evaluation and processing by the DESDM system; a summary is provided in Section 3. These raw single-epoch images are made available by $\mathrm{NOAO}$ and are accessible as described in Section 6.

\subsection{Survey Progress through DRI}

Between Y1 and Y3, 38,850 wide-field exposures passed baseline survey-quality thresholds based on effective exposure time and PSF FWHM (Morganson et al. 2018, Section 4.7) and are included in co-add processing by DESDM (Morganson et al. 2018). The median air mass of DR1 survey-quality exposures was 1.22 , with $>99 \%$ of exposures taken at air mass $<1.4$. Meanwhile, the median delivered seeing (FWHM) was $g=1.12, r=0.96, i=0.88, z=0.84$, and $Y=0$ " 90 (Figure 3). Note that Obs Tac prioritizes observations in the riz bands during periods of good seeing to advance the main science goals of DES (e.g., cosmological constraints from weak gravitational lensing). Figure 4 shows the distribution of sky 


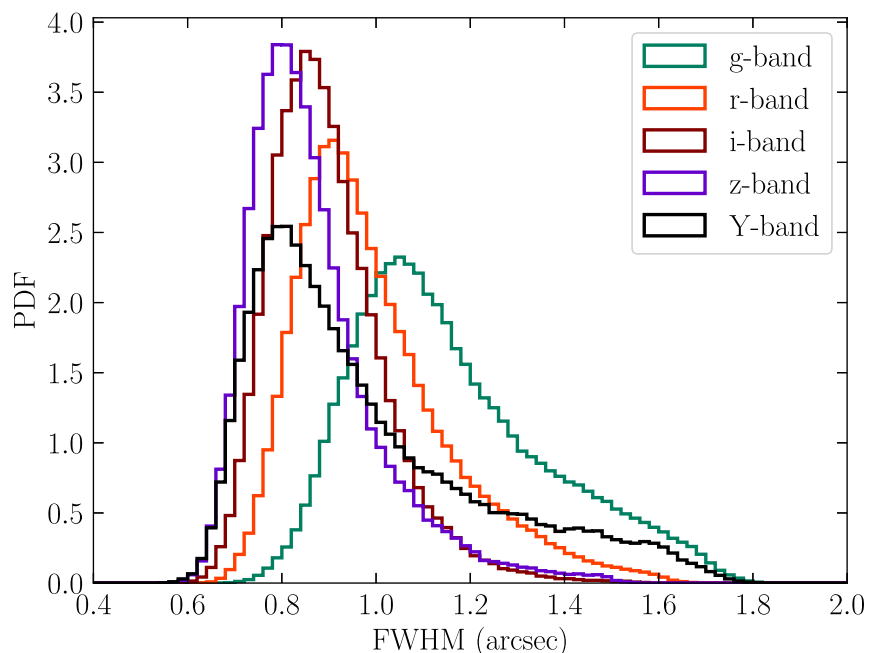

Figure 3. Normalized histograms showing the distribution of PSF FHWM for single-epoch images that form the DR1 co-add.

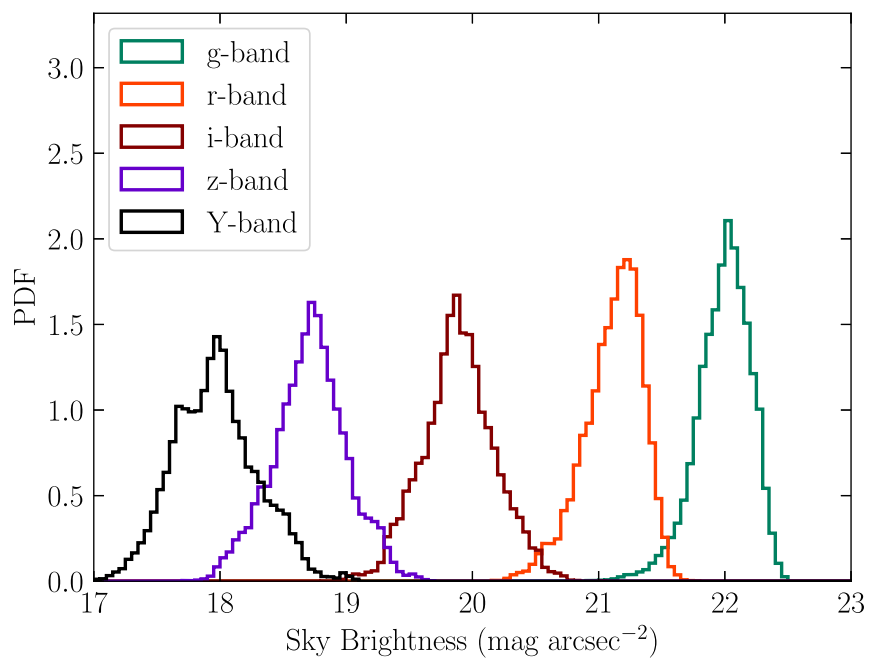

Figure 4. Normalized histograms showing the distribution of sky brightness for single-epoch images that form the DR1 co-add. All magnitudes are given in the $\mathrm{AB}$ system.

brightness levels for single-epoch images; the median sky brightness is $g=22.01, r=21.15, i=19.89, z=18.72$, and $Y=17.96$ mag $\operatorname{arcsec}^{-2}$. The resulting median single-epoch effective image noise level (square root of the calibrated image variance), including additional contributions from read noise and shot noise of the dome flat, is $g=25.25, r=24.94$, $i=24.31, z=23.58$, and $Y=22.28 \mathrm{mag} \mathrm{arcsec}^{-2}$.

Each position in the DES DR1 footprint is typically covered by three to five overlapping DECam exposures in each of the griz $Y$ bands (Figure 5). As an example, a map for the number of overlapping $i$-band exposures across the footprint is shown in Figure 6.

The total sky coverage of DR1 was estimated using maps of the individual image coverage generated by mangle (Hamilton \& Tegmark 2004; Swanson et al. 2008) and converted to HEALPix (Górski et al. 2005) maps with spatial resolution comparable to the size of gaps between individual CCDs (nside = 4096, 0'86; Drlica-Wagner et al. 2018). When requiring at least one exposure in a given band, the areal coverage of each individual band is $g=5224, r=5231$, $i=5230, \quad z=5234$, and $Y=5227 \mathrm{deg}^{2}$. When requiring

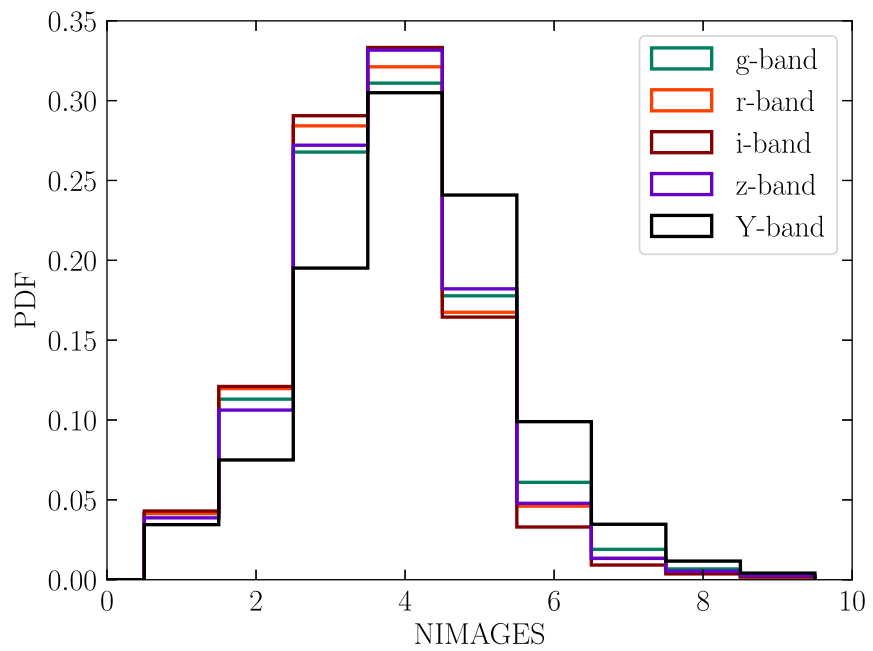

Figure 5. Histograms showing the distribution of overlapping images in each of the grizY bands normalized over the DR1 footprint. Most regions of the footprint are covered with three to five images.

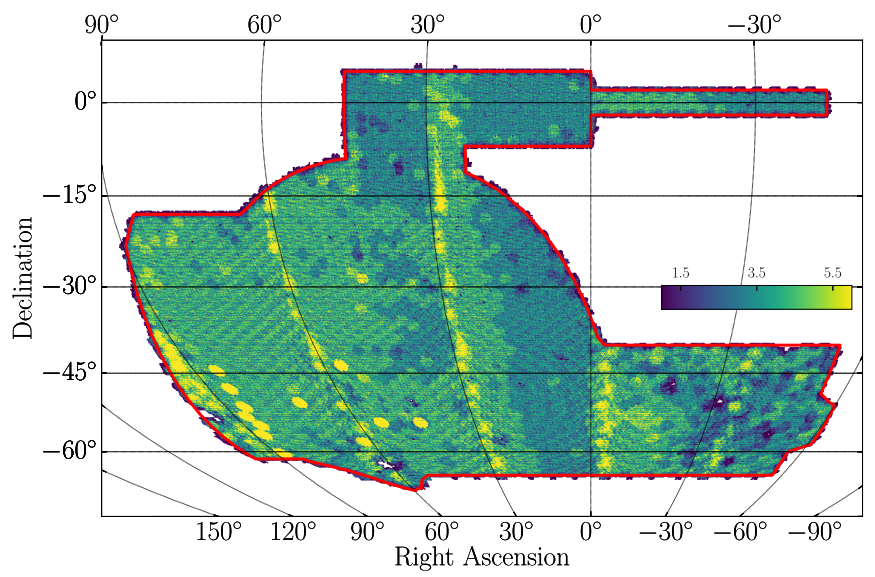

Figure 6. Map of the DES footprint showing the number of overlapping $i$-band exposures. Regions of above-average coverage are a consequence of the DES hexagonal layout scheme and can be found at intervals of $\Delta$ R.A. $=30^{\circ}$. Color range units are number of exposures.

at least one exposure in all of the grizY bands, the DR1 footprint area is $5186 \mathrm{deg}^{2}$. These areal coverage numbers do not account for regions that are masked around bright stars or masked owing to other imaging artifacts, which decrease the areal coverage by $\sim 200 \mathrm{deg}^{2}$. Note also that we are not releasing mangle products for DR1. Instead, we are providing HEALPix indices for all the objects at different resolutions, as well as a tabulated HEALPix map with nside $=32$ for the footprint. See Section 6 for more details about these products.

\section{Data Release Processing}

We briefly describe the DESDM processing pipeline applied to the DES data to generate the DR1 data products. ${ }^{84} \mathrm{DR} 1$ is based on the DESDM Y3A2 internal release to the DES Collaboration, referring to the second annual release of data products obtained from the first three seasons of DES science operations and the Science Verification period. Where possible,

\footnotetext{
${ }^{84}$ Note that the DESDM pipeline differs from the DECam community pipeline.
} 
our pipelines use the AstrOmati $\mathrm{C}^{85}$ suite of tools to perform standard tasks (Bertin \& Arnouts 1996; Bertin et al. 2002; Bertin 2006, 2010, 2011). A full description of the pipeline and the underlying image detrending algorithms can be found in Morganson et al. (2018) and Bernstein et al. (2017a).

\subsection{Single-epoch Processing}

The DES single-epoch processing pipeline (known as "Final Cut") removes instrumental signatures to produce reduced, science-ready images (Morganson et al. 2018). Final Cut performs overscan removal, cross-talk correction, nonlinearity correction, bias subtraction, gain correction, correction for the brighter-fatter effect (Gruen et al. 2015), bad-pixel masking, astrometric matching, flagging of saturated pixels and bleed trails, principal-component background subtraction, secondary flat-field correction, and the masking of cosmic rays and other imaging artifacts. The resulting images from this pipeline form the products that are released through the NOAO Science Archive. ${ }^{86}$ Those images are provided in FITS-formatted files and contain extensions for the science data (SCI), an inversevariance weight (WGT), and a mask of bad pixels (MSK). Note that the weight plane is not altered to account for flagged defects; this allows the user to customize the severity of the defects to be removed based on their own analysis needs. A summary of the flags available is provided in Table 9 of Morganson et al. (2018).

Final Cut also performs PSF model fitting with PSFEx (Bertin 2011) and source detection and measurement with SExtractor (Bertin \& Arnouts 1996). These single-epoch data products are not part of DR1.

\subsection{Multi-epoch (Co-add) Processing}

The multi-epoch pipeline produces co-added images and catalogs of astronomical objects (Morganson et al. 2018). The co-add processing is organized within a tiling scheme that subdivides the sky into square regions with 0.7306 on a side. Co-add images are $10,000 \times 10,000$ pixels with a pixel scale of 0.263 . The choice of images that are tendered as inputs to this pipeline is based on the data quality assessment that occurred in the Final Cut pipeline and the Forward Global Calibration Method (FGCM) for photometric calibration (Burke et al. 2018). In addition, a "blacklist" of images with severe scattered light, ghosts, or bright transient defects (e.g., comets, meteors, and airplanes) is used to exclude additional images from co-add processing. The co-added images are rescaled such that the zero-point is fixed to 30 for all filters. This makes the conversion between flux and magnitude the same for all bands.

The multi-epoch pipeline begins by refining the astrometric solution for the image inputs. This step operates on catalog objects from all input images in all bands simultaneously to provide a consistent alignment between images. The relative astrometry within a tile has a typical rms residual of 30 mas or better. During this process, the absolute astrometry is tied to the Two Micron All Sky Survey (2MASS; Skrutskie et al. 2006). ${ }^{87}$

The next step prepares the images for co-addition. First, the World Coordinate System (WCS; Calabretta \& Greisen 2002; Greisen \& Calabretta 2002) information for each image is

\footnotetext{
85 https://www.astromatic.net/

86 http://archive.noao.edu/

87 The Gaia data releases were not yet available when the processing began.
}

updated to reflect the astrometric shifts solved in the previous step. Then a pair of weight planes are formed, which set the asyet unaltered single-epoch weights to zero to remove defects tracked in the MSK plane. Both weight planes are formed so that we can separately track spatially persistent defects (e.g., saturated stars and bleed trails) and temporary defects (e.g., interpolated bad columns, cosmic rays, satellite trails). The first weight plane contains all defects, while the second weight plane contains only the persistent defects. The Astromatic utility SWarp (Bertin et al. 2002) is then used to form the coadd image (SCI) and weight (WGT) planes; the former uses the first weight plane, while the latter uses the second. A mask plane (MSK) is formed that carries a value of 0 for good pixels and 1 for pixels where no good data exist (due to lack of image coverage or persistent defects).

A subsequent execution of SWarp is then used to form a detection image that is a linear combination of the $r+i+z$ bands using the "CHI-MEAN" weighting (Drlica-Wagner et al. 2018, Appendix B), and PSFEx is used to obtain a PSF model for each tile. Initial catalogs are constructed using SExtractor in dual-image mode where the detection image is used to form the segmentation map of sources prior to extracting measurements from the individual co-add images. We caution that the PSF model is unable to fully account for discontinuities that occur at image boundaries. This limits the precision of the measurements of quantities such as MAG_PSF to no better than a few percent. In addition, morphological classifiers based on the co-add PSF model, such as CLASS STAR and SPREAD_MODEL, can have a degraded/varied performance.

To provide a solution that overcomes most of the limitations of the co-add catalog products, the single-epoch catalogs are matched to the co-add detection catalog, and weighted averages of the single-epoch MAG_PSF and SPREAD_MODEL measurements are made from all unflagged detections of the same object for each band. We add the WAVG_ prefix to indicate these weighted-average quantities (Morganson et al. 2018).

These weighted-average measurements are included among the public data release products. Longer term, the DES Collaboration is pursuing improved photometry through the use of multi-epoch, multiband, and multi-object fitting that operates on the Final Cut single-epoch images (Drlica-Wagner et al. 2018). Those products are maturing but are beyond the scope of the current public data release. They should become available along with the results from the DES Y3 cosmology analyses.

\section{Data Quality}

In this section, we provide a general assessment of the DR1 data quality, including astrometric and photometric precision, imaging depth in terms of measurement $\mathrm{S} / \mathrm{N}$ and object detection completeness, morphological object classification accuracy, and the identification and removal of likely artifacts. A summary of data quality metrics is found in Table 1.

\subsection{Astrometry}

The DR1 astrometric solution is derived in two steps using SCAMP with 2MASS as the reference catalog (Morganson et al. 2018). At the single-epoch stage, we find internal astrometric uncertainties of $g=42, r=36, i=37, z=39$, and $Y=56$ mas, as determined from the median of $2 \mathrm{D}$ 

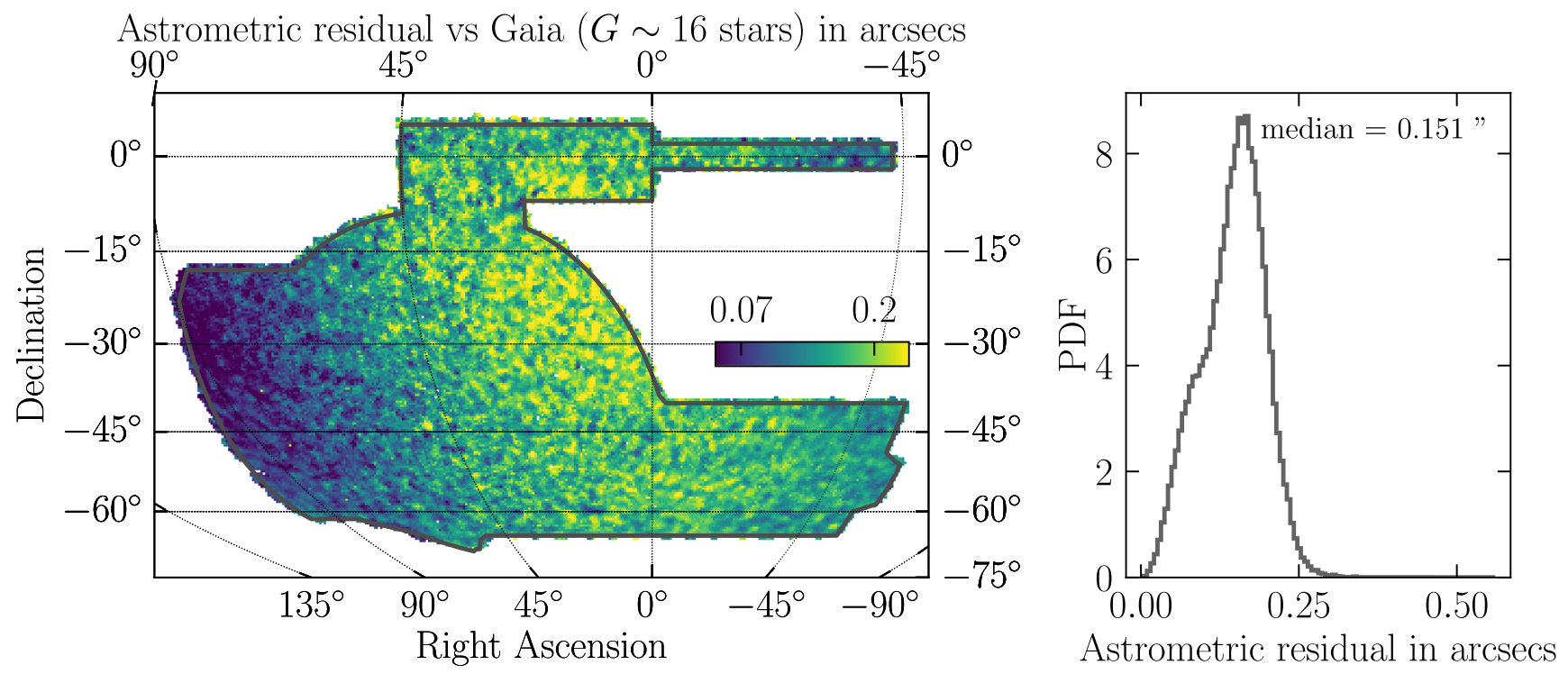

Figure 7. Absolute astrometric precision (total distance) measured relative to stars in Gaia DR1 with $G$-band magnitude, $G_{\text {Gaia }} \sim 16$ (Jordi et al. 2010 ). Left: mean value of the astrometric discrepancy with respect to Gaia vs. sky position computed within HEALPix cells of resolution ns ide $=256$. Right: normalized histogram showing the distribution of astrometric offsets. Color range units are in arcseconds.

Table 1

DES DR1 Key Numbers and Data Quality Summary

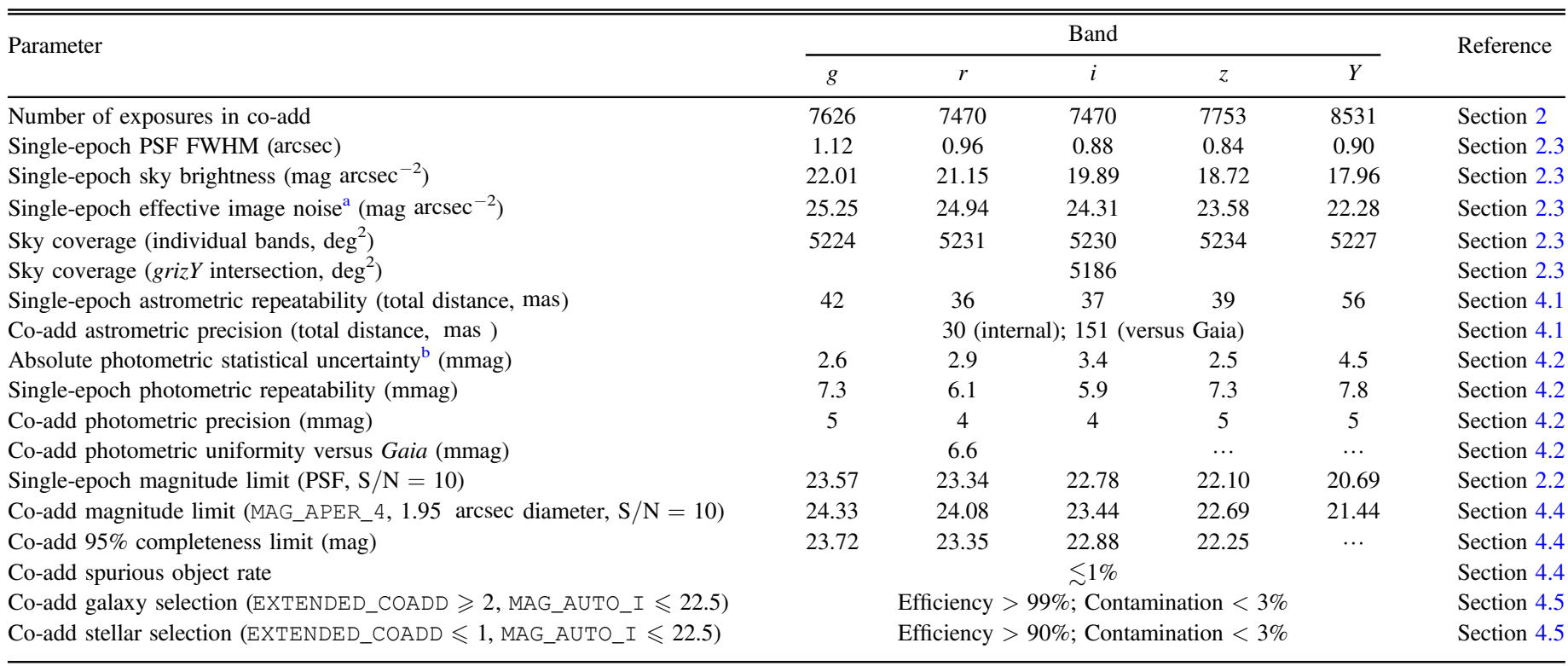

Notes. For parameters representing a distribution, the median or mean values are quoted as specified in the main text. All magnitudes are in the AB system. a Square root of the calibrated image variance, including read noise.

${ }^{\mathrm{b}}$ The Hubble CalSpec standard star C26202 is used as an absolute reference for the AB system.

angular separations between repeated measurements of bright stars from individual exposures. Following the astrometric refinement step for image co-addition by SCAMP, the estimated internal astrometric precision for the co-add is $\sim 30$ mas rms (median over co-add tiles, averaging all five bands). The absolute astrometric uncertainty of the co-add is evaluated with respect to Gaia DR1 (Gaia Collaboration et al. 2016). Figure 7 shows that the 2D astrometric residuals are smallest in regions with larger stellar densities closer to the Galactic plane (for comparison, see Figure 14), and the median over the footprint is $\sim 151$ mas. Further details of astrometric calibration, including ongoing development using Gaia as a reference catalog, are provided in Bernstein et al. (2017b).

\subsection{Photometry}

The relative standard bandpass flux measurements for astronomical sources in DR1 have been calibrated using a forward modeling technique to account for both atmospheric and instrumental components of the total system throughput (FGCM; Burke et al. 2018). The parameters of the model were initially fit from observations taken in photometric conditions to establish a network of calibration stars that spans the full 

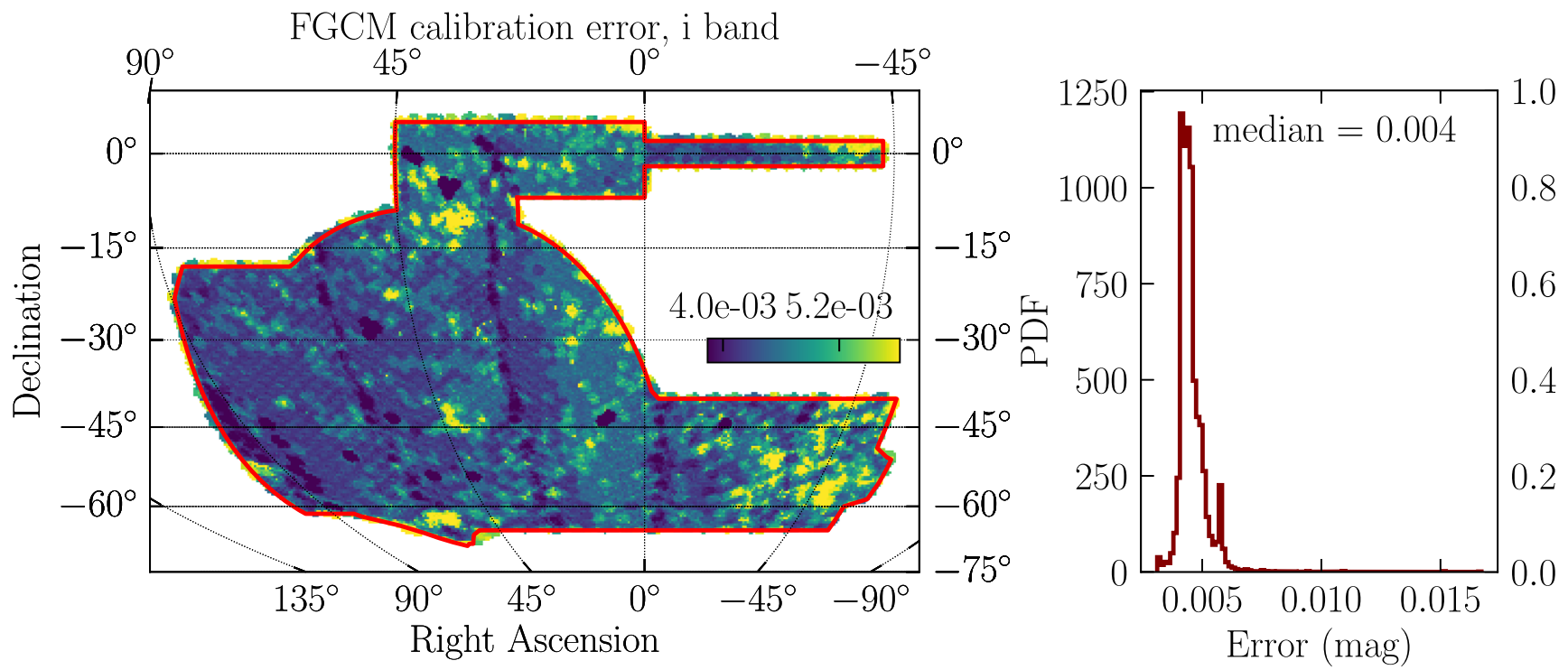

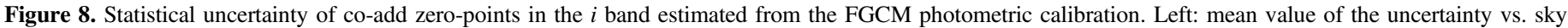

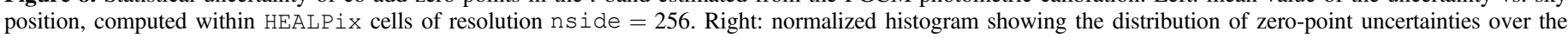
footprint. Color range units are in $\mathrm{AB}$ magnitudes.

survey footprint. This network was subsequently used to refine the calibration of exposures taken in nonphotometric conditions. Meanwhile, the absolute photometric calibration of DR1 is tied to the spectrophotometric Hubble CALSPEC standard star C26202 (Bohlin et al. 2014) located in the SN field C3.

Over 100 repeated measurements of C26202 in a variety of conditions yielded a set of small shifts $(\sim 3 \mathrm{mmag})$ to place the DES photometry on the AB system. These shifts have been pre-applied to the DR1 zero-points. The statistical uncertainty on these shifts is estimated to be $g=2.6, r=2.9, i=3.4$, $z=2.5$, and $Y=4.5 \mathrm{mmag}$. Additional sources of systematic uncertainty on the absolute photometric calibration could arise from uncertainty in the level of out-of-band light leakage and uncertainty in the synthetic photometry of C26202. We are currently undertaking observations and analysis of two additional HST CALSPEC standards to reduce the systematic uncertainty of the $\mathrm{AB}$ offsets.

We reproduce below several of the key results from Burke et al. (2018) regarding the relative photometric calibration. The single-epoch photometric statistical precision (associated with random errors in the FGCM fit parameters) derived from repeated measurements of FGCM calibration stars is $g=7.3$, $r=6.1, \quad i=5.9, \quad z=7.3$, and $Y=7.8 \mathrm{mmag}$. Under the assumption that successive tiled observations of the same fields yield largely independent model fit parameters (as would be expected from the widely spaced observations in DES), we estimate the statistical precision of co-add zero-points by combining the fit results from overlapping exposures. The median co-add zero-point statistical uncertainty is $g=5$, $r=4, i=4, z=5$, and $Y=5 \mathrm{mmag}$. Figure 8 shows an example distribution for the $i$ band. As a validation, we compare the photometric uniformity of DES DR1 to the spacebased Gaia G-band photometry (Figure 9). Variations in uniformity are found to be $6.6 \mathrm{mmag}$, as estimated from a Gaussian fit to the offset distribution between $G_{\text {Gaia }}$ and $G_{\text {pred }}(r)$ predicted from the DES $r$ band (Appendix B).

To account for extinction from interstellar dust, we include an additional column in the DR1 photometry tables for $E(B-V)$ values from the reddening map of
Schlegel et al. (1998, hereafter SFD98) at the location of each catalog object. The $E(B-V)$ values were obtained using a linear interpolation of the zenithal equal-area projected map distributed by SFD98. We computed fiducial interstellar extinction coefficients, $R_{b}$, for each band so that the corrections to the FGCM calibrated source magnitudes are $A_{b}=E(B-V) R_{b}$. Fiducial coefficients are derived using the Fitzpatrick (1999) reddening law with $R_{V}=3.1$ and the Schlafly \& Finkbeiner (2011) calibration adjustment to the original SFD98 reddening map $(N=0.78)$. Note that the Schlafly \& Finkbeiner (2011) calibration adjustment is included in our fiducial reddening coefficients; these coefficients are intended to be used directly with $E(B-V)$ values from the original SFD98 reddening map. We integrate over the DR1 standard bandpasses (Section 5.4) considering a fixed source spectrum that is constant in spectral flux density per unit wavelength, $f_{\lambda}\left(\operatorname{erg~cm}{ }^{-2} \mathrm{~s}^{-1} \AA^{-1}\right)$, and adopted the lowextinction limit. The latter simplification is appropriate for DES, for which $E(B-V)<0.1$ mag over $\sim 99 \%$ of the footprint. The resulting multiplicative coefficient for each band is $R_{g}=3.186, R_{r}=2.140, R_{i}=1.569, \quad R_{z}=1.196$, and $R_{Y}=1.048$. The DES science team continues to explore systematic uncertainties associated with interstellar extinction modeling.

In general, the photometry columns included in the DR1 database tables are not dereddened by default. However, the DR1_MAIN table includes additional columns for the dereddened versions of MAG_AUTO and WAVG_MAG_PSF indicated by a _DERED suffix (Appendix E).

\subsection{Flagged Objects}

For co-add objects, if any pixel is masked in all of the contributing exposures for a given band, the IMAFLAGS_ISO = 1 flag is set for that band. This flag is predominantly set for saturated objects and objects with missing data. We recommend a baseline quality criterion of IMAFLAGS_ISO $=0$ (in the relevant bands) for most science applications since the majority of flagged objects have unreliable photometry. The DES DR1 catalog also includes other standard 

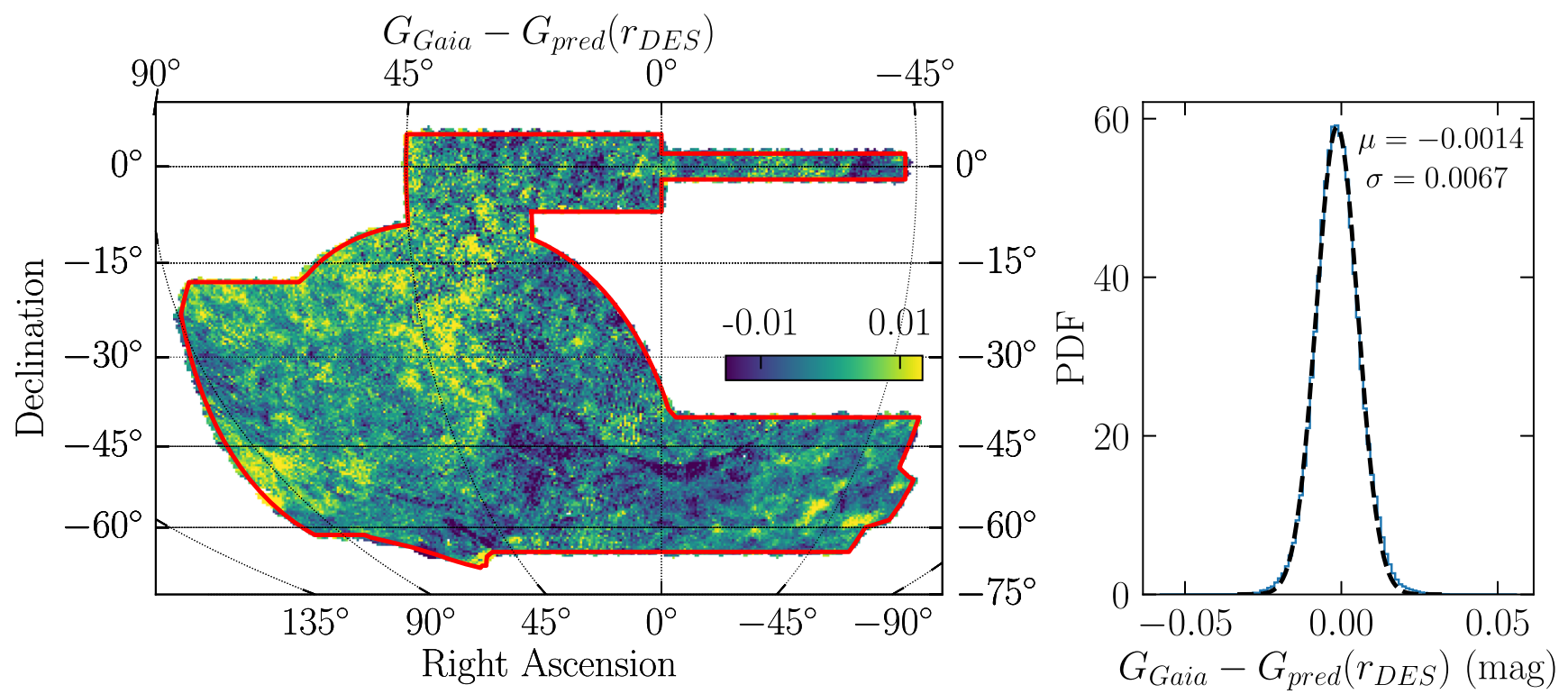

Figure 9. Distribution of photometric residuals against Gaia's $G$ band (mag units) across the DES footprint used to evaluate the uniformity of the co-add FGCM calibration. Left: mean value of this residual vs. sky position in a HEALPix cell of nside $=256$. Right: normalized histogram of photometric residuals over the footprint. Color range units are in $\mathrm{AB}$ magnitudes.

Table 2

DES DR1 Co-add Catalog Median Depth Estimates for the Sample of All High-quality Objects

\begin{tabular}{lcccc}
\hline \hline \multirow{2}{*}{ Method } & \multicolumn{3}{c}{ Band } \\
\cline { 2 - 5 } & $g$ & $r$ & $i$ & 23 \\
\hline Maximum in number counts (MAG_AUTO) & 24.32 & 23.89 & 23.41 & 22.09 \\
Measured with S/N = 10 (MAG_AUTO) & 23.52 & 23.10 & 22.51 & 21.81 \\
Measured with S/N = 10 (MAG_APER_4) & 24.33 & 24.08 & 23.44 & 22.69 \\
Imaging depth from mangle (MAG_APER_4) & 24.282 & 23.952 & 23.335 & 21.61 \\
Detection completeness of 95\% (MAG_AUTO) & 23.72 & 23.35 & 22.88 & 22.25 \\
\hline
\end{tabular}

Note. All magnitudes are given in the AB system.

flags FLAGS provided by the SExtractor pipeline. A summary of SExtractor FLAGS bitmask values and warning descriptions is provided in Appendix D.

\subsection{Depth}

The effective depth of the DES DR1 wide-field co-add catalog is dependent on the photometric measurement of interest and can be quantified through various approaches. Here we derive simple depth estimates from the flux distribution of cataloged objects, the magnitude corresponding to a fixed $\mathrm{S} / \mathrm{N}$ threshold $(\mathrm{S} / \mathrm{N}=10)$, an aperture estimate from the input imaging, and object detection completeness. In general, the type of source and surface brightness must also be considered when evaluating the survey depth.

The settings used for the source extraction and deblending steps of the DESDM pipeline allow for efficient detection of objects with $\mathrm{S} / \mathrm{N} \sim 10$ in the $r+i+z$ composite detection image (Morganson et al. 2018). At the bright end, saturation effects start to become important at $r<16$. For even brighter magnitudes, the fraction of saturated objects increases until objects are no longer cataloged owing to pixel-level masking applied during processing. For each of the depth studies considered here, we selected a sample of high-quality DR1 coadd objects using FLAGS_[GRIZ] $<4$ and IMAFLAGS_ISO_ $[$ GRIZ $]=0$. No star-galaxy selection has been applied to the sample of analyzed objects. Below we describe these different approaches to estimate the depth of DR1; results from each method are provided in Table 2.

\subsubsection{Flux Distribution}

The distribution of astronomical sources is weighted toward low-flux sources. A crude estimate of the detection threshold is given by the mode of the number count distribution of sources as a function of magnitude. Figure 10 shows the number counts of co-add objects as a function of MAG_AUTO in all five DES bands. The mode of the MAG_AUTO distribution is $g=24.32$, $r=23.89, i=23.41, z=22.09$, and $y=21.40$. No restrictions were placed on source morphology (i.e., stars vs. galaxies) for this estimate.

\subsubsection{Magnitude Limit at Fixed Signal-to-noise Ratio}

The magnitude limit corresponding to a fixed $\mathrm{S} / \mathrm{N}$ for a given photometric measurement (e.g., MAG_AUTO) can be empirically determined from the distribution of magnitude uncertainties as a function of magnitude (Rykoff et al. 2015). SExtractor provides an estimate of the photometric uncertainty through the MAGERR quantities, which are estimated from the fluctuations of the background around the sources. These are related to $\mathrm{S} / \mathrm{N}, \delta F / F$, via the differentiation 


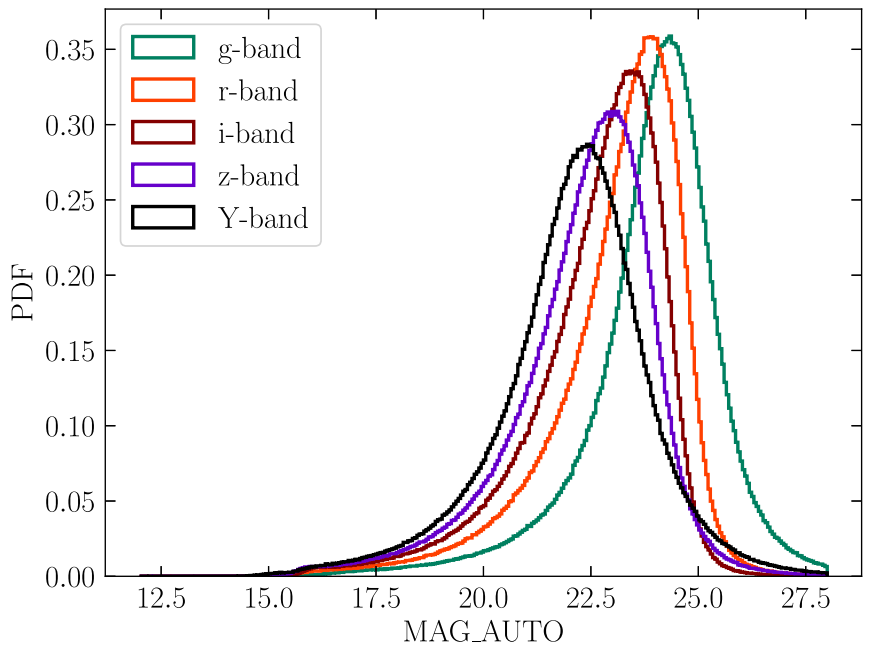

Figure 10. Normalized histograms of source counts binned by SExtractor's MAG_AUTO quantity showing the flux distribution of detected sources. All magnitudes are given in the AB system.

of Pogson's law (Pogson 1856):

$$
\delta m=\frac{-2.5}{\ln 10} \frac{\delta F}{F} .
$$

We summarize in Table 2 the characteristic $\mathrm{S} / \mathrm{N}=10$ thresholds for MAG_AUTO corresponding to $\delta F / F \sim 0.1$ in each of the grizY bands. The left panel of Figure 11 shows the MAG_AUTO distribution for DES DR1 catalog objects with $0.10837<$ magerr_auto $<0.10877$. The spread in these distributions comes from the dependence of $\mathrm{S} / \mathrm{N}$ on source properties (e.g., surface brightness) and survey nonuniformity.

\subsubsection{Depth from Image Properties}

It is also possible to estimate the DES DR1 imaging depth using mangle (Hamilton \& Tegmark 2004; Swanson et al. 2008), which generates a vectorized map of the survey coverage accounting for the focal plane geometry and imaging artifacts (i.e., bright star masks, bleed trails, satellite trails; Drlica-Wagner et al. 2018; Morganson et al. 2018). The mangle processing produces a co-add weight map from a weighted sum of the single-epoch input images. This weight was converted to an $\mathrm{S} / \mathrm{N}=10$ limiting magnitude for a $2^{\prime \prime}$ diameter aperture, corresponding approximately to the MAG_APER_4 quantity measured by SExtractor (for details, see Drlica-Wagner et al. 2018). The median limiting magnitude across the DES footprint is $g=24.282, \quad r=23.952$, $i=23.335, z=22.628$, and $Y=21.383$ (right panel of Figure 11).

\subsubsection{Object Detection Completeness}

Another measure of effective imaging depth is the object detection completeness relative to deeper imaging data. We evaluated the detection completeness of DES DR1 through a comparison to public CFHTLenS data (Erben et al. 2013) using an overlap region centered on (R.A., decl.) $=\left(34^{\circ} .5,-5^{\circ} .4\right)$ consisting of nine CFHTLenS fields in the W1 patch. ${ }^{88}$ The CFHTLens $5 \sigma$ magnitude limit for a $2^{\prime \prime}$ aperture is $g^{\prime}=25.58$,

\footnotetext{
88 CFHTLenS object catalogs and image masks available at http://www. cfhtlens.org/astronomers/data-store.
}

$r^{\prime}=24.88, i^{\prime}=24.54, y^{\prime}=24.71$, and $z^{\prime}=23.46$. To ensure full coverage in both surveys, we restricted the analysis to regions with CFHTLenS image mask value MASK $=0$ and DES coverage fraction $>99 \%$ in the intersection of the griz bands (see Drlica-Wagner et al. 2018). The effective area of overlap in both surveys including masking is $6.0 \mathrm{deg}^{2}$.

Object matching is performed using a $1^{\prime \prime}$ radius, and we require a robust flux measurement in the respective DES band $(15<$ MAG_AUTO $<30)$ that is roughly consistent with that of CFHTLenS (within $1 \mathrm{mag}$ ) for an object to count as "detected" in DES DR1.

The DES DR1 detection efficiency is defined as the fraction of CFHTLenS objects in a given flux interval that has a matched DES object passing the baseline quality cuts listed above, and it is expressed in the DES photometric system using converted flux measurement from CFHTLenS. The resulting detection efficiency curves are plotted in Figure 12. DES DR1 detection efficiencies are only plotted for the magnitude range brighter than the typical $\mathrm{S} / \mathrm{N}=5$ limiting magnitude of CFHTLenS. The $95 \%$ completeness magnitude threshold obtained from this test is $g=23.72, r=23.35, i=22.88$, and $z=22.25$ (Table 1). CFHTLenS does not include comparable $Y$-band coverage.

Using the same baseline quality selection criteria of FLAGS_[GRIZ] $<4$ and IMAFLAGS_ISO_[GRIZ] $=0$, we find that in each of the griz bands, $\lesssim 1 \%$ of DES objects with MAG_AUTO greater than 20 and less than the typical $\mathrm{S} / \mathrm{N}=5$ limiting magnitude of CFHTLenS lack a matched counterpart in CFHTLenS (Figure 12). This suggests that contamination from spurious objects in DES DR1 is also $\lesssim 1 \%$. We expect that some fraction of unmatched DES objects are astrophysical transients or moving objects. Indeed, the spatial distribution of co-add objects with only one single-epoch detection across the grizY bands is concentrated along the ecliptic.

\subsection{Morphological Object Classification}

A basic selection on object size relative to the PSF can be used to separate samples of spatially extended galaxies from point-like stars and quasars. Accurate object classification becomes challenging for ground-based imaging surveys at faint magnitudes, and accordingly, optimal use of morphological, color, and temporal information is an active area of research (e.g., Fadely et al. 2012; Małek et al. 2013; Bertin et al. 2015; Kim et al. 2015; Kim \& Brunner 2017). Several object classification schemes have been applied to DES data for a variety of science cases (e.g., Chang et al. 2015; Reed et al. 2015; Soumagnac et al. 2015; Drlica-Wagner et al. 2018; Sevilla-Noarbe et al. 2018). The most common classification scheme makes use of the SExtractor SPREAD_MODEL, which compares the fit of a local PSF model to a slightly extended exponential disk model (Desai et al. 2012). Below we show an example using SPREAD_MODEL for object classification in a way that is suitable for both stellar and extragalactic science.

We define a new variable EXTENDED_COADD as the independent sum of several Boolean conditions:

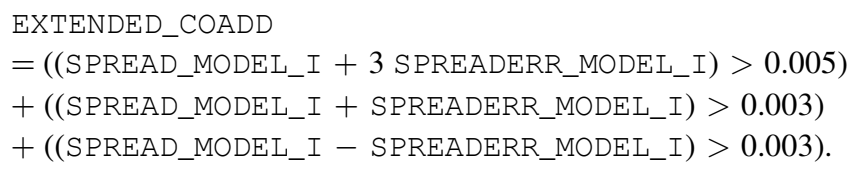



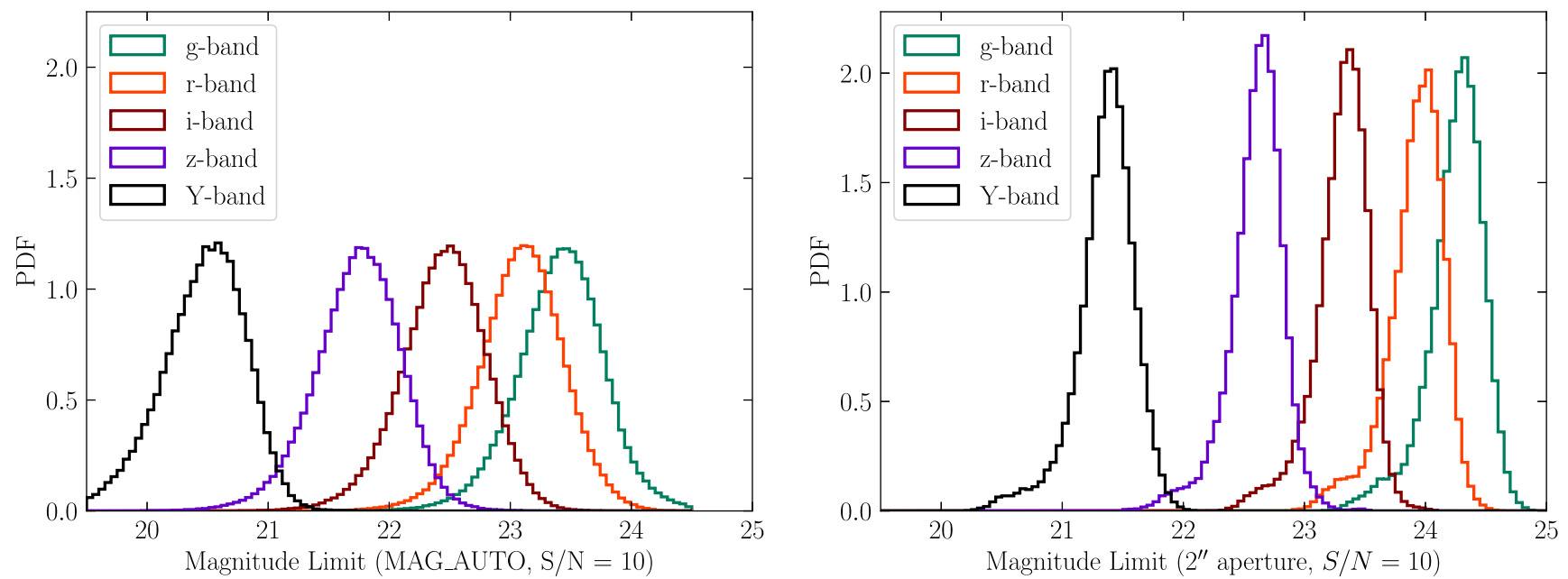

Figure 11. Two estimates of the DES DR1 co-add catalog depth displayed as normalized histograms. Left: catalog depth estimated for MAG_AUTO using catalog objects with $\mathrm{S} / \mathrm{N}=10$ (MAGERR_AUTO $=0.10857$ ). Right: catalog depth for a $2^{\prime \prime}$ aperture estimated from image properties using mangle. All magnitudes are given in the $\mathrm{AB}$ system.

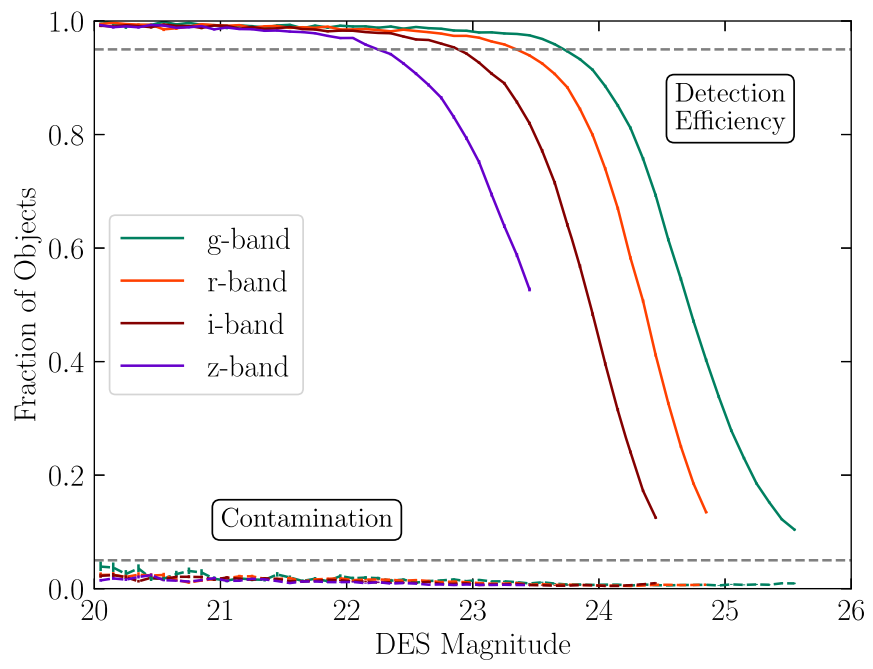

Figure 12. DES DR1 detection efficiency and contamination relative to deeper imaging from CFHTLenS. Solid color curves represent the detection efficiency, while dashed color curves show the fraction of unmatched objects appearing only in DES. For visual reference, gray dashed lines indicate 5\% and $95 \%$ of objects. DES magnitude is given in the AB system.

Note that EXTENDED_COADD is defined by a sequence of Boolean conditions that, when true, add a unit to the classifier. This classifier results in a value of 0 (high-confidence stars), 1 (likely stars), 2 (mostly galaxies), and 3 (high-confidence galaxies).

We evaluate the performance of the example classifier above using three regions in the main body of the DES footprint that overlap fields from HSC-SSP DR1 (Aihara et al. 2018b) with typical seeing in the $i$-band FWHM $\lesssim 0$ !"7: SXDS (Ultra Deep layer), DEEP2_3 (Deep layer), and portions of VVDS (Wide layer). The areal overlap between these HSC-SSP data sets and DES is $\sim 18 \mathrm{deg}^{2}$. The HSCSSP data are of sufficient depth and image quality that a distinct stellar locus is clearly visible in the HSC concentration parameter imag_psf - icmodel_mag to an $i$-band magnitude of $\sim 24.0$. We choose empirically an interval of EXTENDED_COADD values to select stellar or galactic samples with a balance of classification efficiency and purity appropriate for different science cases (Figure 13). Appendix $\mathrm{C}$ demonstrates how this classifier can be used in a number SQL query statement. Figure 14 shows density maps for stars and galaxies selected using EXTENDED_COADD equal to 0 and 3 , respectively. For instance, one can define galaxy (EXTENDED_COADD $>=2$ ) and stellar samples (EXTENDED_COADD $<=1$ ) having $\sim 310$ million and $\sim 80$ million objects, respectively, following the standard object quality selection.

We recommend using quantities based on SExtractor SPREAD_MODEL for morphological classification in DR1. Although the SExtractor quantity CLASS_STAR has been commonly used in the past, we find that SPREAD_MODEL-based classifiers consistently outperform CLASS_STAR, as exemplified by the receiver operating characteristic (ROC) curve shown in Figure 15 and summarized in Table 3. The ROC curves are generated by performing a simple scan of threshold values for each of the SExtractor quantities and using the HSC-SSP classifications described above as a reference. Classifiers based on the weighted-average SPREAD_MODEL from single-epoch detections, WAVG_SPREAD_MODEL, are expected to be more robust for objects that are bright enough to be detected in singleepoch imaging (see Section 4.6). It is expected that classifiers using alternative bands and/or combinations of object measurements will be more appropriate for specific science cases.

\subsection{Known Issues}

The PSF model has limited flexibility to accommodate discontinuities in the effective PSF that can occur in co-added images at boundaries in coverage between individual exposures. In these regions, the local PSF model can fail to accurately fit the point-like sources, and accordingly, both the co-add morphological classifications (i.e., SPREAD_MODEL, CLASS_STAR) and co-add PSF photometry are suspect. The photometry of extended sources is impacted to a lesser degree. Using the $i$ band as an example, we identified such co-add PSF failures by searching for regions with anomalous co-add SPREAD_MODEL_I distributions and estimate that $\lesssim 0.4 \%$ of the footprint is substantially affected. Generally, more robust treatment (especially for point-like sources) is possible with 

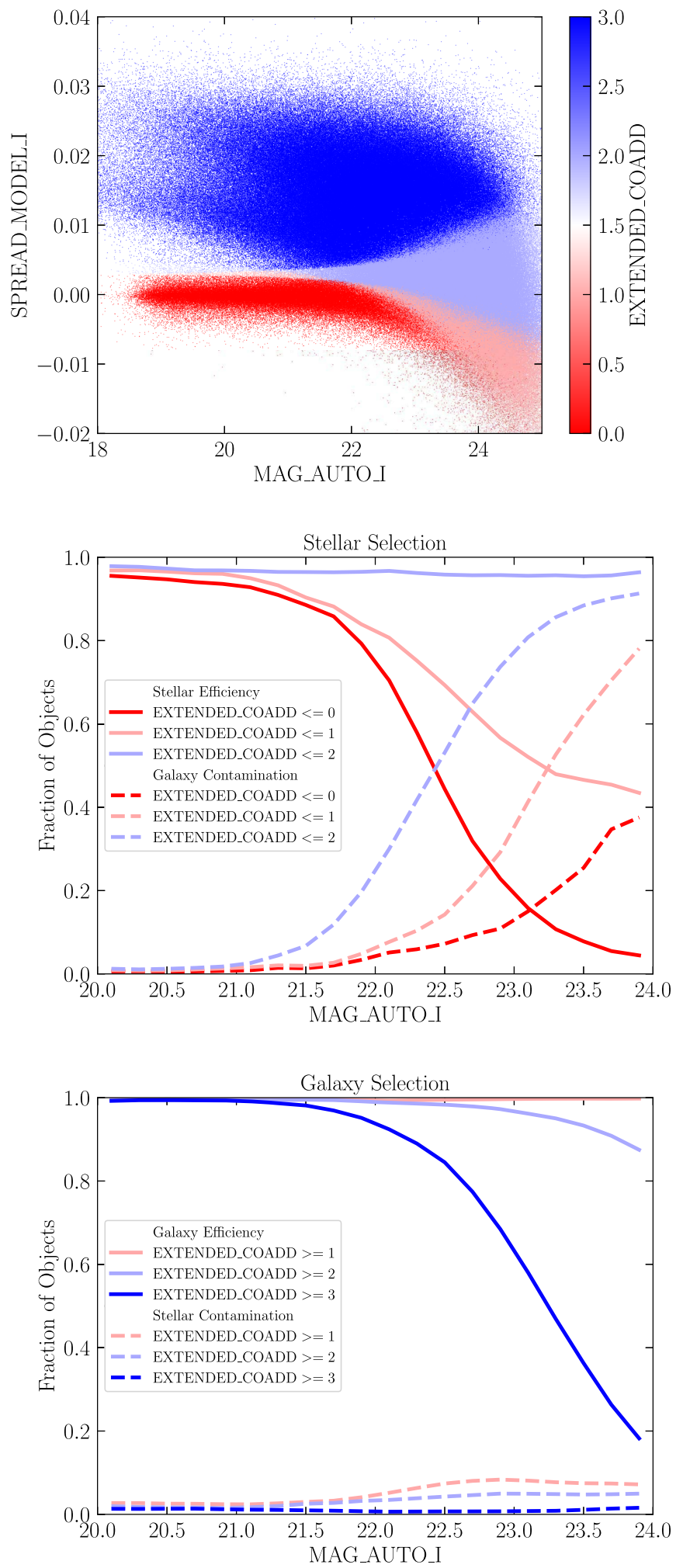

Figure 13. Stars and galaxies occupy distinct regions of spreadmodel-space at bright magnitudes but become more difficult to distinguish at faint magnitudes (top). DES DR1 object classification accuracy vs. HSC-SSP for both stellar (middle) and galaxy (bottom) samples. By using an interval of EXTENDED_ COADD values (Section 4.5), the balance of classification efficiency and purity can be adjusted as appropriate for specific science cases. DES magnitude is given in the $\mathrm{AB}$ system.

weighted-average quantities, at the cost of some loss of object detection completeness for the faintest sources (Figure 16). Due to the known issues in co-add PSF photometry, DES DR1 does not include the co-add MAG_PSF quantities. For studies of point-like sources, we recommend the use of WAVG_MAG_PSF (bright sources) or MAG_AUTO (faint sources).

Among all the objects detected and cataloged, $~ 2.3 \%$ have been flagged by IMAFLAGS_ISO in at least one of the grizY bands, and $\sim 0.1 \%$ have artifacts in all five bands. As described in Section 4.3, it is recommended to use IMAFLAGS_ISO $=0$ as a first filter of clean objects. Most of the objects with IMAFLAGS_ISO $=1$ are saturated stars (Figure 17). A smaller fraction of flagged objects are missing imaging data in one or more of the bands, as shown in Figure 18, and usually have NITER_MODEL $=0$, which is set for objects that did not converge during the photometry measurement.

Scattered light from very bright stars can impact the photometry of nearby objects. Table 4 lists the coordinates and magnitudes of bright stars in the DES footprint. These stars generally cause ragged holes in the imaging coverage of DES DR1 owing to image-level blacklisting of scattered light artifacts (Figure 19). Scattered light from these stars extends beyond image- and catalog-level masking and can be observed at $>1^{\circ}$ in the number counts of objects with extreme colors, i.e., $(g-r)>4$ or $(i-z)>4$ (Drlica-Wagner et al. 2018). Additional care must be taken for analyses close to bright stars.

The DESDM processing pipeline is designed for extragalactic science at high Galactic latitudes. As such, it is not optimized to detect or measure sources in extremely crowded regions. Failures in source deblending are most noticeable in the cores of Galactic globular clusters, where source confusion and saturation reduce the catalog completeness appreciably. Table 5 lists five classical globular clusters within the DES DR1 footprint with integrated $V$-band magnitude brighter than 10 (Harris 1996, 2010 edition). The bottom panels of Figure 19 show two of these clusters, NGC 288 and NGC 1904, where saturation and source confusion can greatly impact source detection and photometric measurements. In addition, nearby bright galaxies (such as NGC 253, NGC 247, NGC 55, and IC 1613) are sometimes deblended into numerous individual objects, and some nearby edge-on spiral galaxies are partially masked.

The tabulated "DES DR1 standard bandpasses" (Figure 1, Section 5.4) included in this release were not the ones used to calculate the zero-points applied to the calibrated images and co-add source catalogs presented in DR1, but instead correspond to an earlier version of the system throughput referred to as the "Y3A1 standard bandpasses." The latter system, which was the one available at the time of data processing, differs from the DES DR1 standard bandpasses in the treatment of the out-of-band system response. The DES DR1 standard bandpasses correct for a small inaccurate representation of $r$-band throughput in the Y3A1 standard bandpasses, which, due to an incorrect input calibration run, considered an unrealistically large light leakage relative to the in-band response $\left(\sim 10^{-2}\right)$ at wavelengths $\sim 8000 \AA$ and $\sim 9200 \AA$. For co-add objects, the impact of setting the outof-band response to zero in the DES DR1 standard bandpasses would lead to a photometry difference of $\lesssim 2 \mathrm{mmag}$ (rms) in the griz $Y$ bands, which is below the level of statistical uncertainty in the co-add zero-points (Section 4.2).

A total of 59.5 of the 62 science CCDs in the DECam focal plane have been fully operational during the DR1 data collection period. CCD 61, which failed in 2012, was not processed for the DR1 data set. Similarly, CCD 2 was not processed between 2013 November and 2016 December 

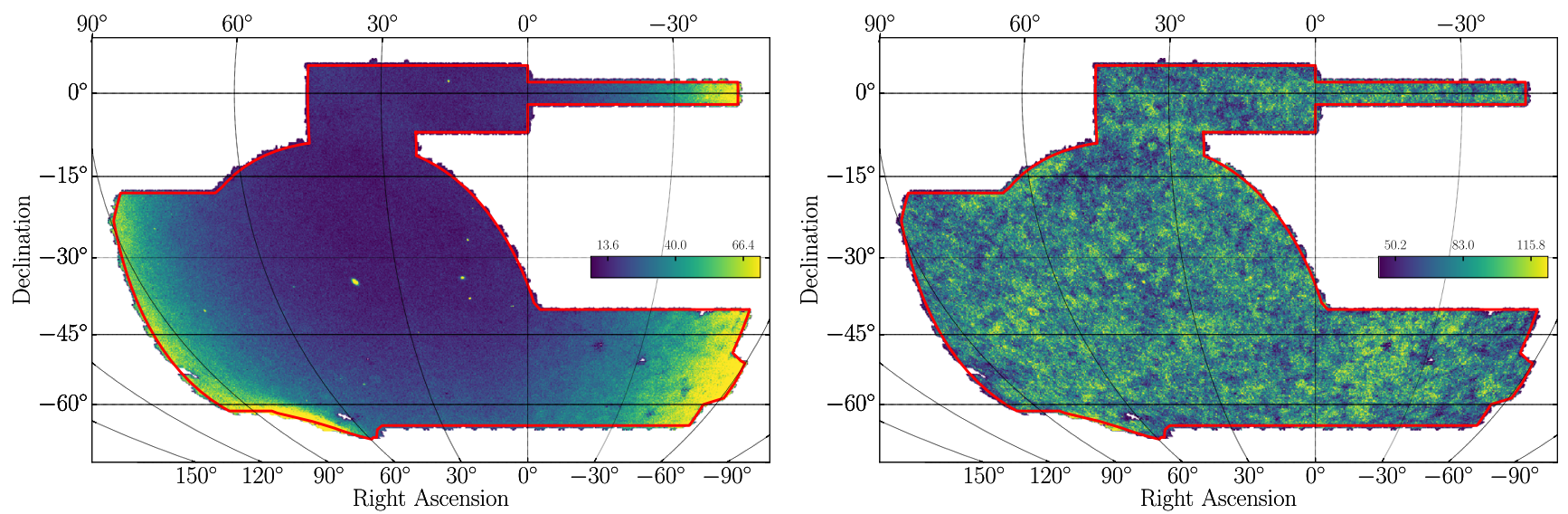

Figure 14. Left: stellar density map at HEALPix nside $=1024$ resolution created with the EXTENDED_COADD $=0$ selection described in Section 4.5 (see also Appendix C). Discrete peaks in the stellar density correspond to globular clusters and dwarf galaxies in the Milky Way halo. Right: analogous galaxy density map created with the EXTENDED_COADD $=3$ selection. Color range units are number of objects per HEALPix nside $=1024$ pixel.

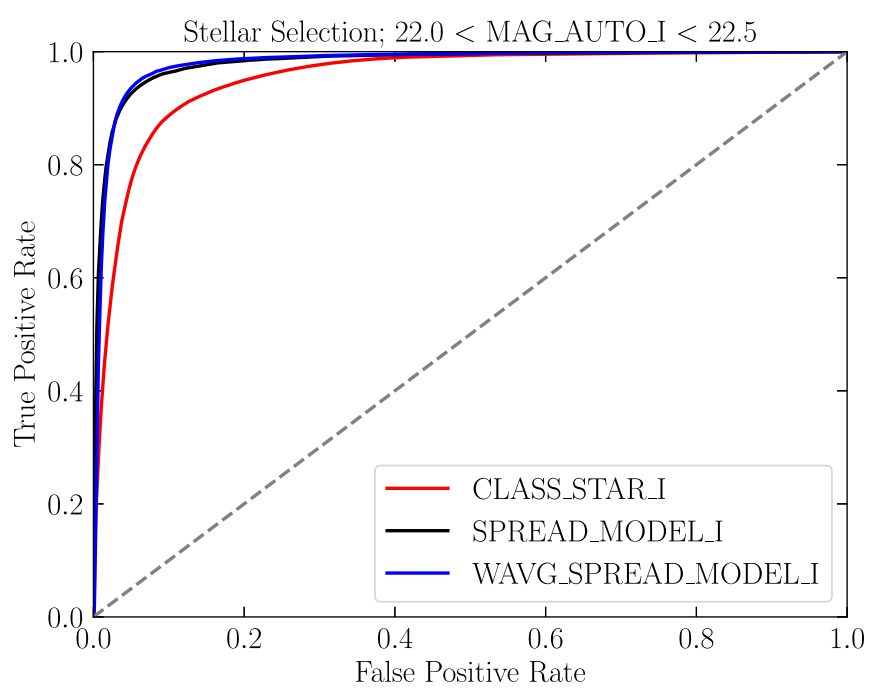

Figure 15. ROC curve for a stellar selection in the magnitude range $22.0<$ MAG_AUTO_I $<22.5$ (AB system). The SPREAD_MODEL outperforms CLASS_STAR in classification accuracy of DES DR1 objects with respect to HSC-SSP. In this case, the SPREAD_MODEL and WAVG_SPREAD_ MODEL variants provide a very similar classification power, as denoted by the closely overlapping black and blue lines, respectively. The diagonal dashed line corresponds to the expectation from a random classifier.

(overlapping with observations included in this release), during which time it was not functional. Amplifier A of CCD 31 has an unpredictable, time-variable gain and is not included in this release. Amplifier B of CCD 31 functions normally and has been included. The rest of the science CCDs are performing within specifications and are usable for science. See Figure 20 for a layout of the DECam focal plane mosaic with the positions of affected CCDs marked.

\section{Release Products}

Here we detail the individual products included in DES DR1. The primary components of DES DR1 are derived from the union of 10,338 co-add tiles covering the DES footprint. The tile distribution for a portion of the SDSS Stripe 82 region is shown in the inset of Figure 2. To view all tiles and a HEALPix map (nside $=32$ ) of 1753 pixels covering the entire DR1, visit https://des.ncsa.illinois.edu/easyweb/footprint.

\subsection{Images}

DES DR1 images can be grouped into two categories:

(a) Calibrated Single Exposures: 38,850 exposures with photometric calibration, corresponding to 4,124,753 individual CCD images. The number of exposures per band is $N_{g}=7626, N_{r}=7470, N_{i}=7470, N_{z}=7753$, and $N_{Y}=8531$. Each raw exposure is $\sim 0.5 \mathrm{~GB}$ in size (compressed). These images can be accessed through NOAO Data Lab. ${ }^{89}$

(b) Co-add Images: As a result of the multi-epoch pipeline described in Section 3, a total of 10,338 tiles of $10 k \times 10 k$ pixels spanning $0.7306 \mathrm{deg}$ on a side at a resolution of $0.263 \operatorname{arcsec}$ pixel $^{-1}$ (see inset of Figure 2) were produced in each of the five bands. These images, along with the $r+i+z$ combined detection image used, are publicly available, with a total of 62,028 images constituting $\sim 11$ TB of data.

\subsection{Catalogs}

The co-add source extraction process detected and cataloged 399,263,026 distinct objects. Morphological object information includes object centroids, shape parameters, HEALP ix indices, and processing flags. Several different photometric measurements and associated uncertainties are provided, including AUTO, PETRO, WAVG_PSF, and assorted aperture magnitudes (Table 6). These measurements are distributed in three database tables: DR1_MAIN, DR1_MAGNITUDE, and DR1_FLUX served from an Oracle database at NCSA. The DR1_MAIN table contains all object information that is not a photometric measurement or uncertainty, augmented by MAG_AUTO and WAVG_MAG_PSF (and associated uncertainties), and interstellarextinction-corrected versions. The other two tables contain auxiliary magnitude and flux measurements (with associated uncertainties) in addition to extra information that is present in all three tables, such as coordinates, flags, and HEALPix indices. We note that these tables contain two sets of coordinates for the objects, namely, (RA, DEC) and (ALPHAWIN J2000, DELTAWIN_J2000), which are computed in the same manner; the difference between them is that $R A$ and

89 http://datalab.noao.edu/ 


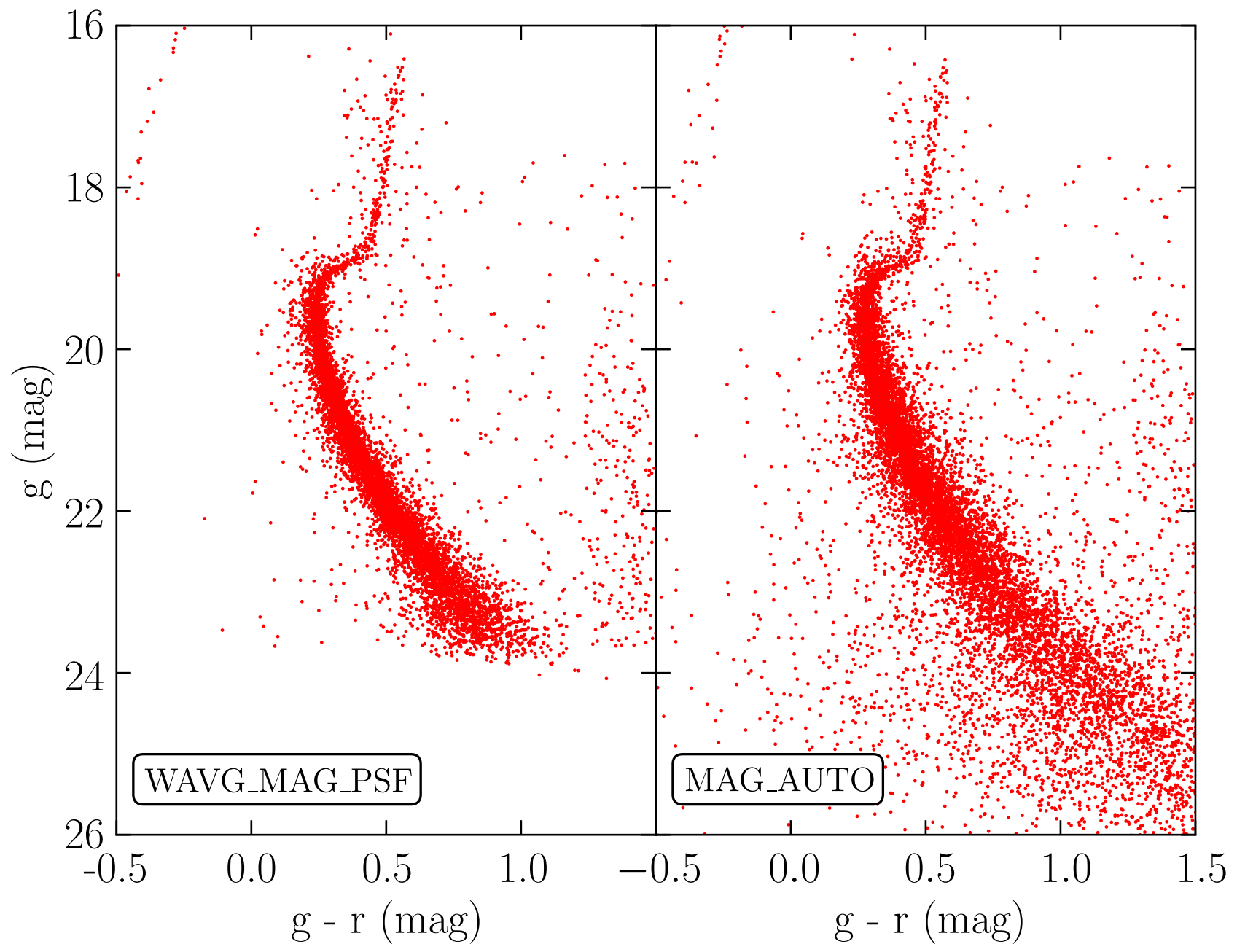

Figure 16. Color-magnitude diagram for a stellar sample selected within a $15^{\prime} \times 15^{\prime}$ box centered on the M2 globular cluster (see Appendix C). The weightedaverage PSF photometry from WAVG_MAG_PSF (left) yields a tighter locus but does not extend as deep as the MAG_AUTO photometry (right). All magnitudes are given in the $\mathrm{AB}$ system.

Table 3

Stellar Classification Accuracy Quantified as the Area under the ROC Curve (AUC) in Three Flux Intervals, Using HSC-SSP as a Reference (see Figure 15)

\begin{tabular}{lcc}
\hline \hline Quantity & WAVG_SPREAD_MODEL & SPREAD_MODEL \\
\hline $21.5<$ MAG_AUTO_I $<22.0$ & 0.994 & 0.991 \\
$22.0<$ MAG_AUTO_I $<22.5$ & 0.981 & 0.981 \\
$22.5<$ MAG_AUTO_I $<23.0$ & 0.917 & 0.948 \\
\hline
\end{tabular}

Note. In this case, the AUC statistic is the probability that the simple classifier will correctly rank a randomly chosen star higher than a randomly chosen galaxy. DES magnitude is given in the $\mathrm{AB}$ system.

DEC are truncated to six decimals in order to provide a search indexing and table partitioning on these columns, while ALPHAWIN_J2000 and DELTAWIN_J2000 are doubleprecision quantities to be used when precise measurements are needed. All spatial-based queries should use RA and DEC in their condition statements. Additionally, the table DR1_TILE_INFO contains information about the processed tiles, such as sky location, geometry, number of objects, and file paths to access the associated images and object catalogs. For a complete description of these tables, we refer the reader to Appendix E.

\subsection{Files}

In order to provide a convenient way to download all the catalog data at once, and noting that the tile is the basic processing unit for the survey, we have created FITS file versions of the catalog tables grouped by co-add tile. This amounts to 31,014 total files (for the DR1_MAIN, DR1_MAGNITUDE, and DR1_FLUX tables) with almost $2.5 \mathrm{~TB}$ of catalog data. Both the catalog and corresponding image file paths can be obtained from the DR1_TILE_INFO table (see Appendix $\mathrm{C}$ for an example) and can be accessed through the interfaces described in Section 6. 

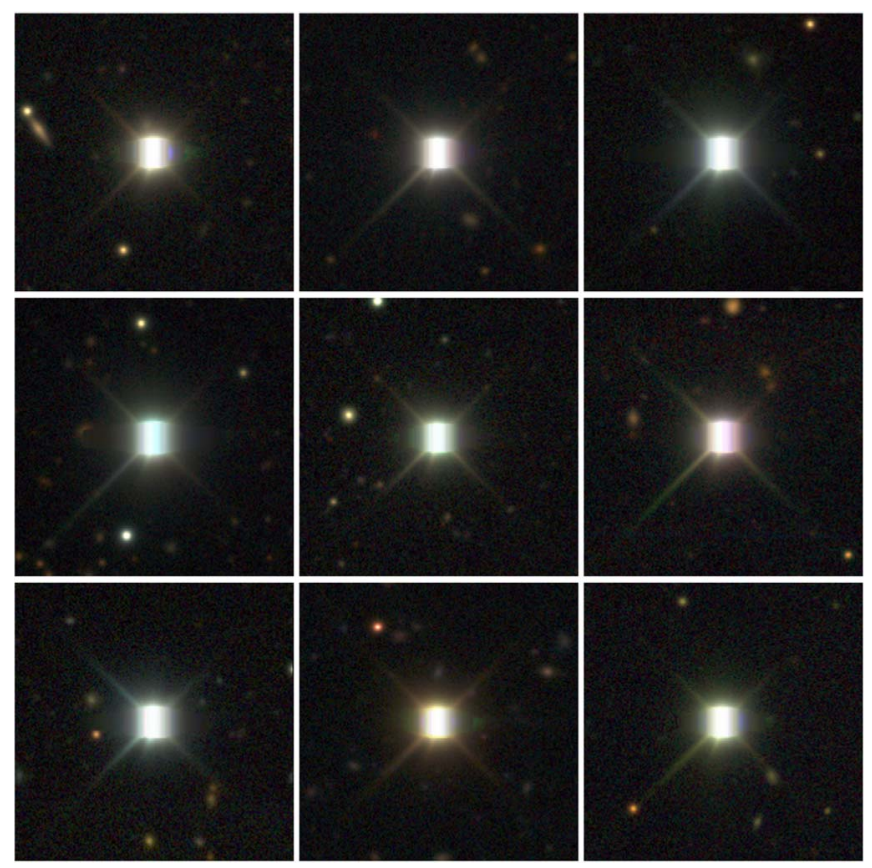

Figure 17. Composite gri $1^{\prime} \times 1^{\prime}$ cutouts of saturated stars with IMAFLAGS_ISO $=1$.

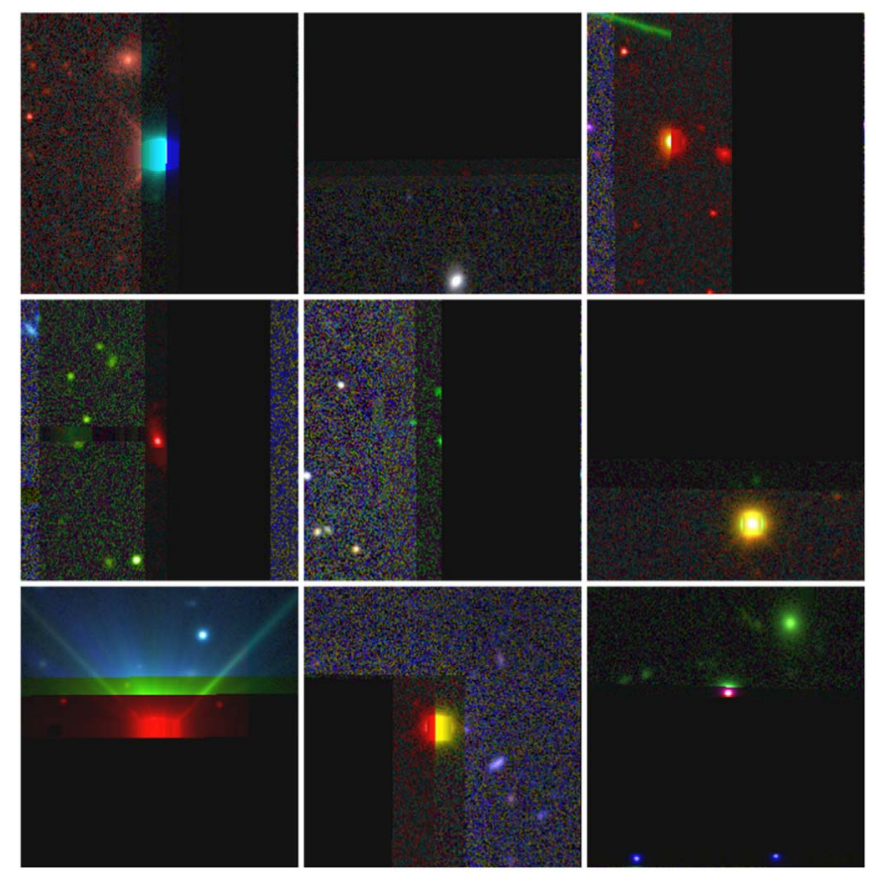

Figure 18. Composite gri $1^{\prime} \times 1^{\prime}$ cutouts of objects with IMAFLAGS_ISO $=1$ and NITER_MODEL $=0$. The majority of such objects, but not all, have missing imaging data.

\subsection{DES DR1 Standard Bandpasses}

DES DR1 includes an updated characterization of the Blanco/ DECam total system response (instrument and atmosphere) for the grizY bands (Figure 1). ${ }^{90}$ The DES DR1 standard bandpasses are defined as the average CCD response across the focal plane as measured with the DECal system (Marshall et al. 2013), together with a standard atmospheric transmission computed with the

\footnotetext{
90 http://www.ctio.noao.edu/noao/content/DECam-filter-information
}
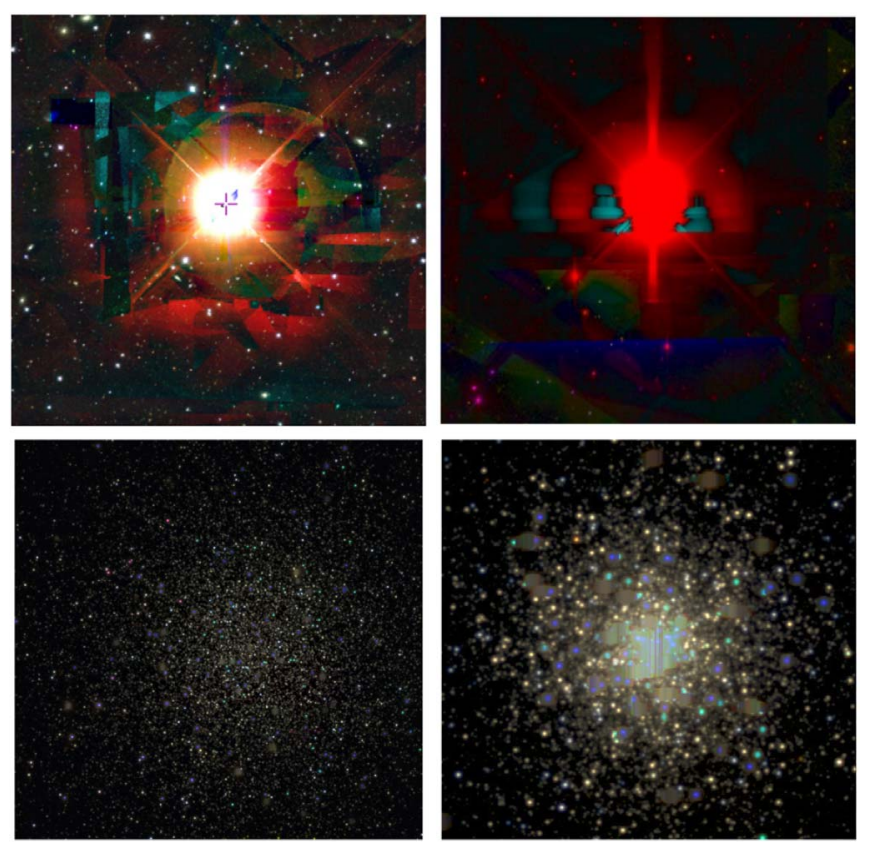

Figure 19. Examples of very bright stars, $R$ Dor $\left(30^{\prime} \times 30^{\prime}\right.$ cutout; top left $)$ and $\alpha$ Phe $\left(14^{\prime} \times 14^{\prime}\right.$ cutout; top right $)$, and globular clusters, NGC $288\left(8^{\prime} \times 8^{\prime}\right.$ cutout; bottom left) and NGC $1904(2$ '.5 $\times 2$ 2.5 cutout; bottom right), found within the DES DR1 footprint.

Table 4

List of Very Bright Stars within the DES Footprint

\begin{tabular}{lccc}
\hline \hline Star & $\begin{array}{c}\text { R.A. } \\
(\mathrm{deg})\end{array}$ & $\begin{array}{c}\text { Decl. } \\
(\mathrm{deg})\end{array}$ & $\begin{array}{c}\text { Apparent Magnitude } \\
(V \mathrm{mag})\end{array}$ \\
\hline$\alpha$ Col (Phact) & 84.9121 & -34.0741 & 2.65 \\
$\alpha$ Phe (Ankaa) & 6.5708 & -42.3061 & 2.38 \\
$\alpha$ Eri (Achernar) & 24.4288 & -57.2367 & 0.46 \\
$\alpha$ Hya (Alphard) & 29.6925 & -61.5697 & 2.00 \\
$\gamma$ Eri (Zaurak) & 59.5075 & -13.5086 & 2.91 \\
$R$ Dor & 69.1900 & -62.0775 & 5.40 \\
$\alpha$ Car (Canopus) & 95.9879 & -52.6958 & -0.74 \\
$\alpha$ Pav (Peacock) & 306.4121 & -56.7350 & 1.94 \\
$\alpha$ Gru (Alnair) & 332.0583 & -46.9611 & 1.74 \\
$\beta$ Gru (Tiaki) & 340.6671 & -46.8847 & 2.15 \\
\hline
\end{tabular}

Table 5

Brightest Globular Clusters within DES DR1

\begin{tabular}{lccc}
\hline \hline Globular Cluster & $\begin{array}{l}\text { R.A. } \\
(\mathrm{deg})\end{array}$ & $\begin{array}{l}\text { Decl. } \\
(\mathrm{deg})\end{array}$ & $\begin{array}{c}\text { Apparent Magnitude } \\
(\mathrm{mag})\end{array}$ \\
\hline NGC 288 & 13.2 & -26.58 & 8.1 \\
NGC 1261 & 48.075 & -55.13 & 8.4 \\
NGC 1851 & 78.525 & -40.05 & 7.3 \\
NGC 1904 & 81.045 & -24.523 & 8.0 \\
NGC 7089 & 323.375 & -0.8167 & 6.5 \\
\hline
\end{tabular}

MODTRAN IV code (Berk et al. 1999) using parameters typical of the environmental conditions encountered during DES observations (for details, see Burke et al. 2017). For example, the air mass adopted for the standard atmosphere, 1.2, is well matched to the median air mass of exposures entering the co-add, 1.22. The system response is defined in steps of $5 \AA$ from $3800 \AA$ to $11000 \AA$. Out-of-band light leakage has been directly measured with DECal to be $\lesssim 10^{-3}$ relative to the in-band 


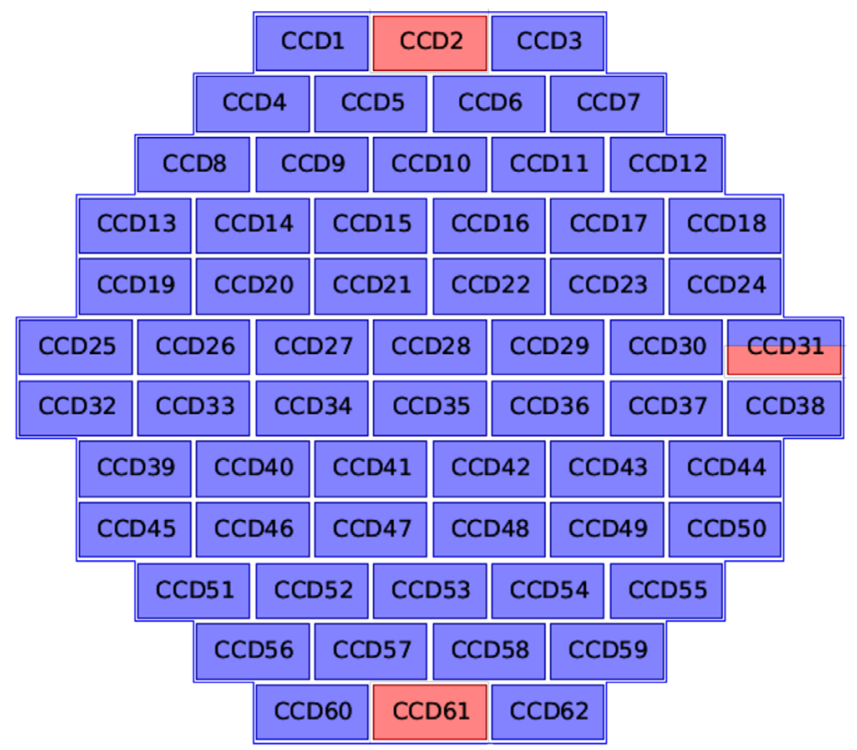

Figure 20. DECam focal plane CCD layout oriented with north at the top and east on the right. The rectangles represent the 62 science CCDs, each of which is divided into two halves oriented along the long direction and read by two amplifiers. CCDs 2 and 61 were inactive for most of the DES data included in DR1. Amplifier A of CCD 31 has time-variable gain and has not been processed for this release. These areas are marked in red.

Table 6

Diameters for the Set of Aperture Magnitudes

\begin{tabular}{lcc}
\hline \hline Column Name & $\begin{array}{c}\text { Diameter } \\
\text { (pixels) }\end{array}$ & $\begin{array}{c}\text { Diameter } \\
\text { (arcsec) }\end{array}$ \\
\hline MAG_APER_1 & 1.85 & 0.49 \\
MAG_APER_2 & 3.70 & 0.97 \\
MAG_APER_3 & 5.55 & 1.46 \\
MAG_APER_4 & 7.41 & 1.95 \\
MAG_APER_5 & 11.11 & 2.92 \\
MAG_APER_6 & 14.81 & 3.90 \\
MAG_APER_7 & 18.52 & 4.87 \\
MAG_APER_8 & 22.22 & 5.84 \\
MAG_APER_9 & 25.93 & 6.82 \\
MAG_APER_10 & 29.63 & 7.79 \\
MAG_APER_11 & 44.44 & 11.69 \\
MAG_APER_12 & 66.67 & 17.53 \\
\hline
\end{tabular}

response, and vendor measurements of witness samples suggest that the out-of-band leakage is typically at the $10^{-5}$ to $10^{-4}$ level. While detailed characterization of the out-of-band response is ongoing, the throughput of the DES DR1 standard bandpasses is defined as zero for out-of-band wavelengths (caveats are mentioned in Section 4.6).

\subsection{Software}

All software used in the DESDM pipelines described in Section 3 and in Morganson et al. (2018) can be accessed from the release page itself $^{91}$ or from the DES Github Organization. $^{92}$ Together with access to the software used to generate these products and the configuration described in Morganson et al. (2018), we provide the main ingredients to reprocess the data in a manner similar to that done by DES.

\footnotetext{
91 https://des.ncsa.illinois.edu/releases/dr1/dr1-docs/processing

92 https://github.com/DarkEnergySurvey
}

\section{Data Access}

Access to the DES DR1 data is provided through a collaborative partnership between NCSA, $^{93}$ LIneA, $^{94}$ and NOAO. ${ }^{95}$ From these institutions, a rich and complementary set of tools and interfaces were developed to access and interact with DES data in different ways that cover a broad set of use cases that enable scientific discovery. In this section we describe the main tools provided to access DES DR $1 .{ }^{96}$

\subsection{NCSA DES access}

NCSA provides the primary set of basic web applications to access DES DR1 data. Developed at NCSA, DESaccess (https://des.ncsa.illinois.edu/easyweb) provides the user with an interface to submit asynchronized jobs to perform SQL queries against an Oracle DB that contains the DES DR1 catalogs and to generate cutouts from a given list of positions from the co-add images. It also contains information regarding the catalogs, an interactive footprint finder to locate positions and tiles, and means to access images and catalogs in a tilebased format. The main services can be summarized as follows:

1. SQL web client: We provide an SQL web client that allows the user to submit asynchronous query jobs against the Oracle 12 database that contains the DES DR1 tables. The submitted jobs enter into a queue, and results can be retrieved at later times in either CSV, FITS (Wells et al. 1981), or HDF5 (The HDF Group 1997) file format, supporting compression in some of the cases. Big jobs are divided into chunks of $1.5 \mathrm{~GB}$ to facilitate file transfer. This interface also provides means to check syntax and to evaluate synchronous jobs for a quick view. Results files are served from an HTTP server that allows file transfer to remote machines. The query used and the results persist for a limited period of time under a "My-Jobs" tab. The query interface is powered using easyaccess (Carrasco Kind et al. 2018), ${ }^{97}$ an enhanced SQL command-line interpreter designed for astronomical surveys and developed for DES.

2. Cutout server: The cutout server allows the user to generate cutouts up to $12^{\prime}$ on a side centered on a given set of positions. The returned files include cutouts in all bands, a cutout in the detection image, a color TIFF image created with STIFF (Bertin 2012) by combining the gri bands, and a PNG image. The typical sizes for these files are $85 \mathrm{MB}$ for the FITS images, $9 \mathrm{MB}$ for the color TIF, and $16 \mathrm{MB}$ for the PNG version, for the case of a $12^{\prime} \times 12^{\prime}$ cutout. These cutout jobs also go into a queue, and results files can be retrieved later and served remotely. The header of the cutouts is a copy from the original header from the images with extra keys indicating the center of the cutout RA_CUTOUT and DEC_CUTOUT. Results are preserved for a limited period and can also be retrieved under a "My-Jobs" tab within the web service. No stitching is performed for objects near the edge of the tile. This feature might be added in the future.

\footnotetext{
93 http://www.ncsa.illinois.edu/

94 http://www.linea.gov.br/

95 https://www.noao.edu/

96 https://des.ncsa.illinois.edu/releases/dr1/dr1-access

97 https://github.com/mgckind/easyaccess
} 
3. Footprint: A lightweight interactive tool that displays the DES footprint and its tiles. This tool can be used to search for a position in the sky and return the corresponding tile information, including name, corner coordinates, and list of files for download. It also allows searches by TILENAME (the tile name identifier) when the name is available. This service also provides access to all the processed tiles, including associated images and catalogs, directly from the file server.

The DESaccess code ${ }^{98}$ and the release page ${ }^{99}$ are open sourced. The front end was developed using web components, HTML, JS, and Polymer, ${ }^{100}$ which allows the reuse of alreadyexisting elements. The back end was mainly developed using the Python Tornado web-framework. ${ }^{101}$ The submission jobs are handled by Celery, ${ }^{102}$ which is a distributed queue manager written in Python. All of these applications are containerized using Docker, ${ }^{103}$ and all the node resourcing and scheduling, as well as all the deployment, are managed by Kubernetes, ${ }^{104}$ which allows us to run, manage, and scale containerized applications in a robust and efficient manner.

\subsection{LIneA Science Server}

The images and catalogs generated by DESDM for DR1 can also be accessed by an interface developed by the Laboratorio Interinstitucional de e-Astronomia (LIneA) that supports the participation of Brazilian scientists in DES. It has been designed to offer ways to examine both images and catalogs, compare these with the results of other surveys, and examine the results of queries to the database. The interface consists of the following three services:

1. Sky/Image viewer: The Sky/Image viewer combination integrates third-party tools to allow the user to visualize the entire sky map produced by DES (Aladin Light ${ }^{105}$ developed by $\mathrm{CDS}^{106}$ ) in the form of an HIPs color-coded image, as well as each individual tile using VisiOmatic (Bertin et al. 2015). The native functions, such as zoom, have been augmented with (1) an image layer that allows the display of other surveys; (2) a position locator; (3) the possibility of sharing a display with other users; (4) a map viewer that allows the display of HEALpix maps generated during the processing of the data (e.g., a map of the number of images available at each point in the sky); (5) different grids and polylines, including the original co-add tiles and the border of the DES footprint; and (6) access to the image of a specific tile, which can be examined using Visiomatic.

The image viewer can be used to examine the tile as a whole and to inspect a specific position on the sky in more detail using the native functions of the tool that include snapshot, profile overlays, contrast settings, color mix, zoom, full-screen mode, and catalog overlay. The

\footnotetext{
98 https://github.com/mgckind/desaccess

99 https://github.com/mgckind/des_ncsa

100 https://www.polymer-project.org/

101 http://www.tornadoweb.org/en/stable/

102 http://www.celeryproject.org/

103 https://www.docker.com/

104 https://kubernetes.io/

105 http://aladin.u-strasbg.fr/AladinLite/

106 http://cdsportal.u-strasbg.fr/
}

interface also allows side-by-side comparison of the same sky region using different settings. Other useful functions are the ability to switch on/off markers, recenter the display to provide a visualization of the entire tile, crop part of the image, and download the fits images and catalog associated with the tile being displayed.

Finally, one can also overlay object catalogs, which are classified into three categories: (1) targets-list of positions or objects either uploaded or created using the Target viewer and User Query services described below; (2) object catalog-the DES DR1 object catalog produced by DESDM; and (3) external catalogs-a sample of catalogs available in Vizier. To facilitate the comparison between catalogs, the user can change the symbol, color, and size of each catalog it selects to display.

2. Target Viewer: This service enables the user to examine a list of uploaded positions of objects selected using the User Query service described below. The first page provides a summary of the available target lists, the ability to upload/delete lists, and the ability to mark favorite lists. Adding a list can be done by pasting a list of (R.A., decl.) coordinates. Once a list is selected, the image surrounding an object/position can be visualized by selecting an entry in the list and the corresponding position. The user can then select the columns to be shown, sort according to a given attribute, and comment, rank, and reject an entry. One can also apply a filter, and the filtered list can be downloaded (as CSV or FITS file) or saved for future use.

3. User Query: This service provides access to the database table storing the DES DR1 data from which SQL queries can be written, validated, and executed. The resulting table is displayed under "My Tables," where it can be renamed, a few lines of its content listed, and deleted. Objects selected can be immediately viewed in the Target Viewer after the columns of the resulting table are properly associated with those recognized by the tool.

Given the suite of functionalities available in each tool, tutorials in the form of videos have been prepared to help introduce first-time users to the services. Information about the current limitations is available in the help associated with each tool.

\subsection{NOAO Data Lab}

The NOAO Data Lab (Fitzpatrick et al. 2016) is one of the access portals for DES DR1. The goal of the Data Lab is to enable efficient exploration and analysis of large data sets, including catalogs, with a particular focus on surveys using DECam and the NOAO Mosaic cameras. Among its features are a database for catalog data, accessible both from a Table Access Protocol $\left(\mathrm{TAP}^{107}\right.$ ) service and from direct PostgreSQL queries, web-based, command-line and programmatic catalog and image query interfaces (both custom and via standard VO protocols); remote storage space for personal database and files; a JupyterHub-based notebook analysis environment; and a Simple Image Access (SIA) service. The Data Lab hosts the DES DR1 catalog tables using a PostgreSQL v9.6 database on the back end. The data are identical to those hosted by the DES

107 http://ivoa.net/documents/TAP 
Collaboration through the NCSA portal, but with a few additions: First, the Data Lab database contains tables with cross-matches to other large catalogs (AllWISE, GALEX, HSC, and Simbad) and a table of neighboring objects within a 30 arcsec radius for all objects in DES. Second, the main table contains a few extra columns, such as ecliptic and Galactic coordinates, an $\mathrm{HTM}^{108}$ (Hierarchical Triangular Mesh) index, two supplementary HEALPix indices in RING (nside $=256$ ) and NESTED (nside = 4096) schemes, and precomputed colors. Finally, the data are clustered and indexed using the Q3C scheme (Koposov \& Bartunov 2006), allowing Q3C functions to perform fast spatial queries on the tables.

\subsubsection{Data Lab Services}

1. Both anonymous and authenticated access to DES DR1 through Data Lab services. Anonymous users of the Data Lab can query the DES DR1 database and use the webbased tools. By creating and logging into an account through datalab.noao.edu, authenticated users get access to a dedicated Jupyter notebook server with permanently stored notebooks and $1 \mathrm{~TB}$ of storage space for personal database tables and files.

2. Access to DES DR1 through TAP. The Data Lab exposes the DES DR1 catalog through a TAP service, which may be accessed through a web query form and schema browser. Alternately, users may query DES DR1 through TOPCAT (Taylor 2005) by pointing to the URL of the Data Lab TAP service (https://datalab. noao.edu/query.php?name $=$ tap_schema.schemas) from within the application.

3. Python and command-line query clients. The Data Lab client package (https://github.com/noao-datalab/datalabclient) contains the multipurpose datalab command-line interface and the queryClient Python module. Both interfaces allow synchronous queries from a personal computer, for which control is suspended until a result is returned, using either ADQL (the native TAP interface query language) or the Postgres SQL syntax. The user may also run asynchronous ADQL queries, for which the query operation is given a job ID and run in the background, through the TAP service. The queryClient module is preinstalled on the Data Lab Jupyter notebook server.

4. An image cutout service. The Data Lab SIA service provides access to cutouts of the DES DR1 images. For a given position on the sky, the SIA service returns a table of metadata of all images that fall within the specified radius. The metadata include select header information for each image, along with a URL to retrieve a cutout of a specified size.

5. A JupyterHub notebook server and compute environment. This server provides access to common Python libraries, as well as all Data Lab Python modules, including the authClient authorization module, the queryClient query module, the storeClient virtual storage module, other interface modules, and multiple examples. The Jupyter notebook server provides a convenient way to run code close to the data.

\footnotetext{
${ }^{108}$ http://www.skyserver.org/htm
}

More information on using these services is available on the Data Lab web page: http://datalab.noao.edu.

\section{Summary and Future Releases}

DES has provided a deep view of the south Galactic cap with precise griz $Y$ photometry that will ultimately reach $\sim 24$ th magnitude in the $i$ band over $\sim 5000 \mathrm{deg}^{2}$. We have described here an overview of the survey, data acquisition, and processing pipelines and have given more details on the release products, the data validation, the known issues, and the data access services for the first major public release (DR1).

This release is composed of the reduced images and widefield co-add source catalogs from the first $3 \mathrm{yr}$ of full science operations, consisting of almost 39,000 single exposure images and close to 62,000 co-add images (including all bands and detection images) covering 10,338 tiles over the DES footprint, resulting in nearly 400 million distinct cataloged objects. Benchmark galaxy and stellar samples contain $\sim 310$ million and $\sim 80$ million objects, respectively, following a basic object quality selection.

The primary attributes of DES DR1 are summarized in Table 1, and the data products can be accessed from several complementary platforms hosted at NCSA, NOAO, and LIneA, available at https://des.ncsa.illinois.edu/releases/dr1. The overall high quality and homogeneity of the release will provide a rich legacy for the international astrophysics community (e.g., Dark Energy Survey Collaboration et al. 2016).

DES finishes its scheduled observations in early 2019, and we expect that the next major public DES data release (DR2) will be based on the products available after the survey is completed.

Funding for the DES Projects has been provided by the U.S. Department of Energy, the U.S. National Science Foundation, the Ministry of Science and Education of Spain, the Science and Technology Facilities Council of the United Kingdom, the Higher Education Funding Council for England, the National Center for Supercomputing Applications at the University of Illinois at Urbana-Champaign, the Kavli Institute of Cosmological Physics at the University of Chicago, the Center for Cosmology and Astro-Particle Physics at the Ohio State University, the Mitchell Institute for Fundamental Physics and Astronomy at Texas A\&M University, Financiadora de Estudos e Projetos, Fundação Carlos Chagas Filho de Amparo à Pesquisa do Estado do Rio de Janeiro, Conselho Nacional de Desenvolvimento Científico e Tecnológico and the Ministério da Ciência, Tecnologia e Inovação, the Deutsche Forschungsgemeinschaft, and the Collaborating Institutions in the Dark Energy Survey.

The Collaborating Institutions are Argonne National Laboratory, the University of California at Santa Cruz, the University of Cambridge, Centro de Investigaciones Energéticas, Medioambientales y Tecnológicas-Madrid, the University of Chicago, University College London, the DES-Brazil Consortium, the University of Edinburgh, the Eidgenössische Technische Hochschule (ETH) Zürich, Fermi National Accelerator Laboratory, the University of Illinois at Urbana-Champaign, the Institut de Ciències de l'Espai (IEEC/CSIC), the Institut de Física d'Altes Energies, Lawrence Berkeley National Laboratory, the Ludwig-Maximilians Universität München and the 
associated Excellence Cluster Universe, the University of Michigan, the National Optical Astronomy Observatory, the University of Nottingham, The Ohio State University, the University of Pennsylvania, the University of Portsmouth, SLAC National Accelerator Laboratory, Stanford University, the University of Sussex, Texas A\&M University, and the OzDES Membership Consortium.

Based in part on observations at the Cerro Tololo InterAmerican Observatory, National Optical Astronomy Observatory, which is operated by the Association of Universities for Research in Astronomy (AURA) under a cooperative agreement with the National Science Foundation.

The DES data management system is supported by the National Science Foundation under grant nos. AST-1138766 and AST-1536171. The DES participants from Spanish institutions are partially supported by MINECO under grants AYA2015-71825, ESP2015-66861, FPA2015-68048, SEV2016-0588, SEV-2016-0597, and MDM-2015-0509, some of which include ERDF funds from the European Union. IFAE is partially funded by the CERCA program of the Generalitat de Catalunya. Research leading to these results has received funding from the European Research Council under the European Union's Seventh Framework Program (FP7/20072013), including ERC grant agreements 240672, 291329, and 306478.

Members of LIneA would like to acknowledge the financial support of the special program INCT do e-Universo.

This work made use of the Illinois Campus Cluster, a computing resource that is operated by the Illinois Campus Cluster Program (ICCP) in conjunction with the National Center for Supercomputing Applications (NCSA) and is supported by funds from the University of Illinois at UrbanaChampaign.

This research is part of the Blue Waters sustained-petascale computing project, which is supported by the National Science Foundation (awards OCI-0725070 and ACI-1238993) and the state of Illinois. Blue Waters is a joint effort of the University of Illinois at Urbana-Champaign and its National Center for Supercomputing Applications.

We acknowledge support from the Australian Research Council Centre of Excellence for All-sky Astrophysics (CAASTRO), through project no. CE110001020, and the Brazilian Instituto Nacional de Ciência e Tecnologia (INCT) e-Universe (CNPq grant 465376/2014-2).

This manuscript has been authored by Fermi Research Alliance, LLC, under contract no. DE-AC02-07CH11359 with the U.S. Department of Energy, Office of Science, Office of High Energy Physics. The United States Government retains and the publisher, by accepting the article for publication, acknowledges that the United States Government retains a nonexclusive, paid-up, irrevocable, worldwide license to publish or reproduce the published form of this manuscript, or allow others to do so, for United States Government purposes.

Facility: Blanco (DECam).

Software: SExtractor (Bertin \& Arnouts 1996), PSFEx (Bertin 2011), scamp (Bertin 2006), swarp (Bertin 2010), mangle (Hamilton \& Tegmark 2004; Swanson et al. 2008), HEALPix (Górski et al. 2005), ${ }^{109}$ astropy (Astropy Collaboration et al. 2013), matplotlib (Hunter 2007),

109 http://healpix.sourceforge.net numpy (Van Der Walt et al. 2011), scipy (Jones et al. 2001), healpy, ${ }^{110}$ fitsio, ${ }^{11}$ ngmix (Sheldon 2014), ${ }^{112}$ easyaccess, skymap.

\section{Appendix A DES Terminology}

1. Image: a single raw output file corresponding to 1 of the 62 science CCDs in the DECam focal plane.

2. Hex: a hexagonal field on the sky nominally covered by the 62 science CCDs of a single DECam pointing.

3. Exposure: a collection of 62 science CCD images corresponding to a single pointing of DECam.

4. Tiling: a collection of DECam pointings that covers the DES footprint with minimal gaps and overlaps in a single filter.

5. Tile: a sky area unit used by DESDM to parcel the DES footprint and organize the co-add outputs. Each tile is 0.7306 on a side.

6. Single-epoch: relates to the collection and analysis of individual exposures for a single band prior to coaddition.

7. Co-add: the process of combining the data from multiple exposures over an area in order to increase depth.

8. Y3A2 Release: second annual internal release by DESDM to the DES Collaboration of data products obtained from the first three seasons of DES science operations and the Science Verification period. DR1 is based on Y3A2.

\section{Appendix B Photometric Transformations}

We provide empirical photometric transformations between DES and other surveys that were used for the validation of DR 1 data products.

\section{B.1. Gaia}

A sample of stars taken from the deep SN fields with at least 25 observations in each of the $g, r$, and $i$ bands were matched to Gaia stars with $G<20$ mag . The Gaia $G$-band magnitudes predicted from from DES photometry are

$$
\begin{aligned}
G= & r-0.100+0.150(g-i)-0.013(g-i)^{2} \\
& -0.035(g-i)^{3},
\end{aligned}
$$

valid for stars with $0.3<g-i<3.0$.

\section{B.2. CFHTLenS}

As described in Section 4.4, we transformed photometry from the CFHTLenS survey to the DES system to report the detection efficiency of CFHTLenS objects as a function of magnitude in the DES system. The corresponding transformation equations, which were empirically derived from matched stars with high

\footnotetext{
110 https://github.com/healpy/healpy

111 https://github.com/esheldon/fitsio

112 https://github.com/esheldon/ngmix
} 
measured $\mathrm{S} / \mathrm{N}$, are

$$
\begin{aligned}
g_{\text {DES }} & =g_{\text {CFHTLenS }}+0.040(g-r)_{\text {CFHTLenS }}+0.143 \\
r_{\text {DES }} & =r_{\text {CFHTLenS }}-0.083(g-r)_{\text {CFHTLenS }}+0.089 \\
i_{\text {DES }} & =i_{\text {CFHTLenS }}-0.179(i-z)_{\text {CFHTLenS }}+0.132 \\
z_{\text {DES }} & =z_{\text {CFHTLenS }}-0.067(i-z)_{\text {CFHTLenS }}+0.115 .
\end{aligned}
$$

\section{Appendix C Example Queries}

We provide several example SQL queries to the DR1 Oracle database tables. Note that these queries can be easily modified to ADQL language used by the TAP service at NOAO Data Labs.

1. Sample of objects: This query returns a sample of $0.0001 \%$ of objects from DR1.

SELECT ra, dec, mag_auto_g FROM dr1_main SAMPLE (0.0001);

also, one can select the first rows of a table with a command like

SELECT ra, dec, mag_auto_g FROM dr1_main WHERE ROWNUM $<=10$.

2. Select stars from the M2 globular cluster: This query uses the EXTENDED_COADD example morphological classifier to separate point-like stars from spatially extended galaxies (see Section 4.5). An interstellar reddening correction has been pre-applied to the columns marked with the _dered suffix (see Section 4.2). This query was used to generate Figure 16.

SELECT

$$
\begin{aligned}
& \text { coadd_object_id, } \\
& \text { ra, dec, ebv_sfd98, } \\
& \text { mag_auto_g_dered, } \\
& \text { mag_auto_r_dered, } \\
& \text { wavg_mag_psf_g_dered, } \\
& \text { wavg_mag_psf_r_dered }
\end{aligned}
$$

FROM

WHERE

$$
\text { dr1_main }
$$

$323.36+0.125$

$$
\text { ra BETWEEN } 323.36 \quad-125 \text { AND }
$$

AND dec BETWEEN -0.82 - 0.125

AND $-0.82+0.125$

AND ( (CASE WHEN spread_model_i +3 . * spreaderr_model_i $>0.005$ THEN 1 ELSE 0 END) +

(CASE WHEN spread_model_i + 1.* spreaderr_model_i $>0.003$ THEN 1 ELSE 0 END) +

(CASE WHEN spread_model_i1. * spreaderr_model_i $>0.003$ THEN 1 ELSE O END) ) $<=1$

AND spread_model_i BETWEEN

-0.05 AND 0.05

AND imaflags_iso_g $=0$

AND imaflags_iso_r $=0$

AND flags_g $<4$

AND flags_r $<4$.

3. Create galaxy density map: Create a HEALPix (NEST schema, celestial coordinates) galaxy density map at resolution $\mathrm{nside}=1024(\sim 3$ ! 4):

SELECT

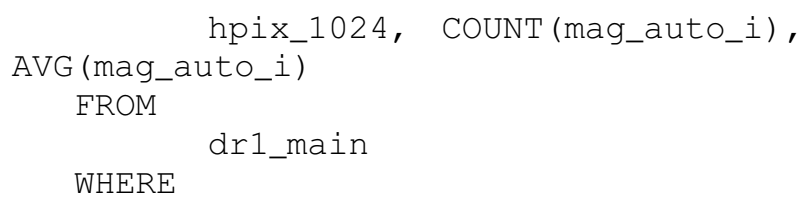

( (CASE WHEN spread_model_i $+3 . *$ spreaderr_model_i $>0.005$ THEN 1 ELSE 0 END) +

(CASE WHEN spread_model_i + 1.* spreaderr_model_i $>0.003$ THEN 1 ELSE 0 END) +

(CASE WHEN spread_model_i-1. * spreaderr_model_i $>0.003$ THEN 1 ELSE 0 END) ) $=3$

$$
\begin{aligned}
-0.05 \text { AND } & 0.05 \\
& \text { AND imaflags_iso_i }=0 \\
& \text { AND flags_i }<4 \\
& \text { AND mag_auto_i }<23
\end{aligned}
$$$$
\text { AND spread_model_i BETWEEN }
$$

GROUP BY

$$
\text { hpix_1024. }
$$

4. Return URLs for the complete co-add object catalogs and grizY co-add images within a small patch of sky:

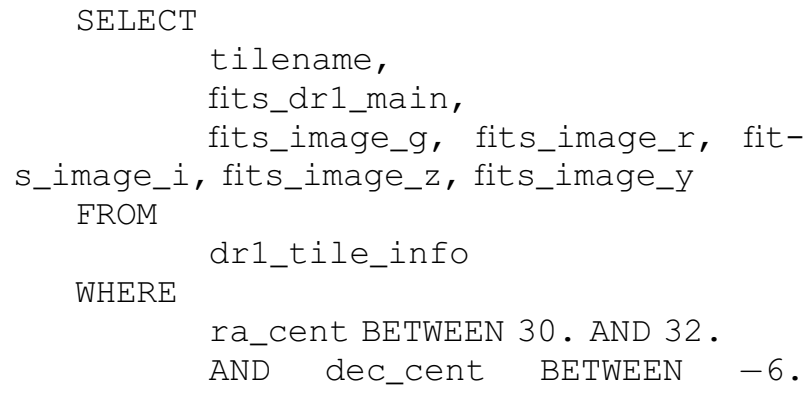

\begin{tabular}{|c|c|}
\hline Bit & Description \\
\hline \multirow[t]{2}{*}{1} & $\begin{array}{l}\text { The object has neighbors, bright and close enough to significantly } \\
\text { bias the }\end{array}$ \\
\hline & $\begin{array}{l}\text { MAG AUTO photometry, or bad pixels (more than } 10 \% \text { of the inte- } \\
\text { grated area affected) }\end{array}$ \\
\hline 2 & The object was originally blended with another one \\
\hline 4 & At least one pixel of the object is saturated (or very close to) \\
\hline 8 & The object is truncated (too close to an image boundary) \\
\hline 16 & Object's aperture data are incomplete or corrupted \\
\hline 32 & Object's isophotal data are incomplete or corrupted \\
\hline 64 & A memory overflow occurred during deblending \\
\hline 128 & A memory overflow occurred during extraction \\
\hline
\end{tabular}

\section{Appendix D SExtractor Flags}

Table 7 summarizes the standard warning flags provided by SExtractor, encoded in the FLAGS bitmask column for each band.

Table 7

Summary of Bitmask Values and Warning Descriptions for the SExtractor FLAGS Column ${ }^{\mathrm{a}}$

Note.

${ }^{a}$ Table data obtained from https://www.astromatic.net/pubsvn/software/ sextractor/trunk/doc/sextractor.pdf. 


\section{Appendix E Released Tables}

The DR1 catalog data are mostly composed of four tables (Tables 8-11). DR1_MAIN includes all the main quantities extracted from the co-add pipeline (Table 8) and important information about the objects. That table also includes MAG_AUTO and WAVG_ MAG_PSF, as well as the corresponding dereddened magnitudes.
DR1_FLUX and DR1_MAGNITUDE contain 15 different measurements of the fluxes and magnitudes (Tables 9 and 10, respectively) for each object. Additionally, these three tables share some commonly used columns to facilitate queries (by avoiding the need to join multiple tables). The fourth table, DR1_TILE_INFO (Table 11), contains information relevant to the processed tiles, from the tile geometry to the URLs of associated files.

Table 8

DR1_MAIN Table Description: 399,263,026 Rows; 213 Columns

\begin{tabular}{|c|c|c|}
\hline Column Name & Description & $\begin{array}{l}\text { Number of } \\
\text { Columns }\end{array}$ \\
\hline COADD_OBJECT_ID & Unique identifier for the co-added objects & 1 \\
\hline TILENAME & Identifier of each one of the tiles on which the survey is gridded & 1 \\
\hline RA & $\begin{array}{l}\text { Right ascension, with quantized precision for indexing (ALPHAWIN_J2000 has full } \\
\text { precision } \\
\text { but not indexed) (deg) }\end{array}$ & 1 \\
\hline ALPHAWIN_J2000 & Right ascension for the object, J2000 in ICRS system (full precision but not indexed) (deg) & 1 \\
\hline DEC & $\begin{array}{l}\text { Declination, with quantized precision for indexing (DELTAWIN_J2000 has full precision but } \\
\text { not indexed) (deg) }\end{array}$ & 1 \\
\hline DELTAWIN_J2000 & $\begin{array}{l}\text { Declination for the object, J2000 in ICRS system (full precision but not } \\
\text { indexed) (deg) }\end{array}$ & 1 \\
\hline GALACTIC_L & Galactic longitude (deg) & 1 \\
\hline GALACTIC_B & Galactic latitude (deg) & 1 \\
\hline XWIN_IMAGE & $X$-centroid from windowed measurements on co-added image (pixels) & 1 \\
\hline YWIN_IMAGE & $Y$-centroid from windowed measurements on co-added image (pixels) & 1 \\
\hline XWIN_IMAGE_G,R,I,Z,Y & $X$-centroid from windowed measurements on co-added band image (pixels) & 5 \\
\hline YWIN_IMAGE_G,R,I,Z,Y & $Y$-centroid from windowed measurements on co-added band images (pixels) & 5 \\
\hline X2WIN_IMAGE_G,R,I,Z,Y & Second moment in $x$-direction, from converged windowed measurements (pixel2) & 5 \\
\hline ERRX2WIN_IMAGE_G,R,I,Z,Y & $\begin{array}{l}\text { Uncertainty in second moment of } x \text {-distribution centroid, from converged windowed mea- } \\
\text { surements (pixel2) }\end{array}$ & 5 \\
\hline Y2WIN_IMAGE_G,R,I,Z,Y & Second moment in $y$-direction, from converged windowed measurements (pixel2) & 5 \\
\hline ERRY2WIN_IMĀGE_G,R,I,Z,Y & $\begin{array}{l}\text { Uncertainty in second moment of } y \text {-distribution centroid, from converged windowed mea- } \\
\text { surements (pixel2) }\end{array}$ & 5 \\
\hline XYWIN_IMAGE_G,R,I,Z,Y & Second moment in $x y$-direction, from converged windowed measurements (pixel2) & 5 \\
\hline ERRXYWIN_IMAGE_G,R,I,Z,Y & $\begin{array}{l}\text { Uncertainty in second moment of } x y \text {-distribution, from converged windowed measurements } \\
\text { (pixel2) }\end{array}$ & 5 \\
\hline HPIX_32,64,1024,4096,16384 & Healpix identifier for its nside grid size, in a NESTED schema & 5 \\
\hline NEPOCHS_G,R,I,Z,Y & Number of epochs the source is detected in single-epoch images & 5 \\
\hline NITER_MODEL_G,R,I,Z,Y & Number of iterations in model-fitting photometric measurements & 5 \\
\hline ISOAREA_IMAGE_G,R,I,Z,Y & Isophotal area of the co-added source (pixel2) & 5 \\
\hline A_IMAGE & Major-axis size based on an isophotal model (pixels) & 1 \\
\hline ERRA_IMAGE & Uncertainty in major-axis size, from isophotal model (pixels) & 1 \\
\hline AWIN_IMAGE_G,R,I,Z,Y & Major-axis size, from second-order windowed moment measurements (pixels) & 5 \\
\hline ERRAWIN_IMĀGE_G,R,I,Z,Y & $\begin{array}{l}\text { Uncertainty in major-axis size, from converged windowed measurement, assuming uncorre- } \\
\text { lated noise (pixels) }\end{array}$ & 5 \\
\hline B_IMAGE & Minor-axis size based on an isophotal model (pixels) & 1 \\
\hline ERRB_IMAGE & Uncertainty in minor-axis size, from isophotal model (pixels) & 1 \\
\hline BWIN_IMAGE_G,R,I,Z,Y & Minor-axis size, from second-order windowed moment measurements (pixels) & 5 \\
\hline ERRBWIN_IMAGE_G,R,I,Z,Y & $\begin{array}{l}\text { Uncertainty in minor-axis size, from converged windowed measurement, assuming uncor- } \\
\text { related noise (pixels) }\end{array}$ & 5 \\
\hline THETA_J2000 & Position angle of source in J2000 coordinates, from nonwindowed measurement (deg) & 1 \\
\hline ERRTHETA_IMAGE & Uncertainty in source position, from isophotal model (deg) & 1 \\
\hline THETAWIN_IMAGE_G,R,I,Z,Y & Position angle of source, for converged windowed measurement grow from $x$ to $y$ (deg) & 5 \\
\hline ERRTHETAWIN_IMAGE_G,R,I,Z,Y & Uncertainty in source position angle, from converged windowed measurement (deg) & 5 \\
\hline FWHM_IMAGE_G,R,I,Z,Y & $\begin{array}{l}\text { FWHM measured from the isophotal area, from elliptical growth curve, modeled in two } \\
\text { dimensions (pixels) }\end{array}$ & 5 \\
\hline FLUX_RADIUS_G,R,I,Z,Y & $\begin{array}{l}\text { Half-light radius for the object, from elliptical growth curve, modeled in two dimensions } \\
\text { (pixels) }\end{array}$ & 5 \\
\hline KRON_RADIUS & Kron radius measured from detection image (pixels) & 1 \\
\hline KRON_RADIUS_G,R,I,Z,Y & Kron radius measured from co-added image (pixels) & 5 \\
\hline CLASS_STAR_G,R,IIZ,Y & $\begin{array}{l}\text { Simple morphological extended source classifier. Values between } 0 \text { (galaxies) and } 1 \text { (stars). } \\
\text { SPREAD MODEL exhibits better performance for morphological classification. }\end{array}$ & 5 \\
\hline
\end{tabular}


Table 8

(Continued)

\begin{tabular}{|c|c|c|}
\hline Column Name & Description & $\begin{array}{c}\text { Number of } \\
\text { Columns }\end{array}$ \\
\hline SPREAD_MODEL_G,R,I,Z,Y & $\begin{array}{l}\text { Morphology based classifier based on comparison between a PSF versus exponential-PSF } \\
\text { model. } \\
\text { Values closer to } 0 \text { correspond to stars, larger values correspond to galaxies }\end{array}$ & 5 \\
\hline SPREADERR_MODEL_G,R,I,Z,Y & $\begin{array}{l}\text { Uncertainty in morphology based classifier based on comparison between PSF versus } \\
\text { exponential-PSF model. }\end{array}$ & 5 \\
\hline WAVG_SPREAD_MODEL_G,R,I,Z,Y & SPREAD MODEL using the weighted-average values from single-epoch detections & 5 \\
\hline WAVG_SPREADERR_MODEL_G,R,I,Z,Y & $\begin{array}{l}\text { Uncertainty in SPREAD MODEL using the weighted-average values from single-epoch } \\
\text { detections }\end{array}$ & 5 \\
\hline FLUX_AUTO_G,R,I,Z,Y & Aperture-flux measurement, elliptical model based on the Kron radius (ADU) & 5 \\
\hline FLUXERR_AUTO_G,R,I,Z,Y & Uncertainty in aperture-flux measurement, elliptical model based on the Kron radius (ADU) & 5 \\
\hline WAVG_FLUX_PSF_G,R,I,Z,Y & Weighted-average flux measurement of PSF fit single-epoch detections (ADU) & 5 \\
\hline WAVG_FLUXERR_PSF_G,R,I,Z,Y & Uncertainty of weighted-average flux measurement of PSF fit single-epoch detections (ADU) & 5 \\
\hline MAG_AUTO_G,R,I,Z,Y & Magnitude estimation, for an elliptical model based on the Kron radius (mag) & 5 \\
\hline MAGERR_AUTO_G,R,I,Z,Y & Uncertainty in magnitude estimation, for an elliptical model based on the Kron radius (mag) & 5 \\
\hline MAG_AUTO_G,R,I,Z,Y_DERED & $\begin{array}{l}\text { Dereddened magnitude estimation (using SFD98), for an elliptical model based on the Kron } \\
\text { radius (mag) }\end{array}$ & 5 \\
\hline WAVG_MAG_PSF_G,R,I,Z,Y & Weighted-average magnitude, of PSF fit single-epoch detections (mag) & 5 \\
\hline WAVG_MAGERR_PSF_G,R,I,Z,Y & Uncertainty of weighted-average magnitude, of PSF fit single-epoch detections (mag) & 5 \\
\hline WAVG_MAG_PSF_G,R,I,Z,Y_DERED & $\begin{array}{l}\text { Dereddened weighted-average magnitude (using SFD98) from PSF fit single-epoch detec- } \\
\text { tions (mag) }\end{array}$ & 5 \\
\hline EBV_SFD98 & $E(B-V)$ reddening coefficient from Schlegel, Finkbeiner \& Davis, 1998 (mag) & 1 \\
\hline BACKGROUND_G,R,I,Z,Y & Background level, by CCD-amplifier (mag) & 5 \\
\hline FLAGS_G,R,I,Z,Y & $\begin{array}{l}\text { Additive flag describing cautionary advice about source extraction process. Use less than } 4 \text { for } \\
\text { well-behaved objects }\end{array}$ & 5 \\
\hline IMAFLAGS_ISO_G,R,I,Z,Y & Flag identifying sources with missing/flagged pixels, considering all single-epoch images & 5 \\
\hline
\end{tabular}

Table 9

DR1_FLUX Table Description: 399,263,026 Rows, 179 Columns

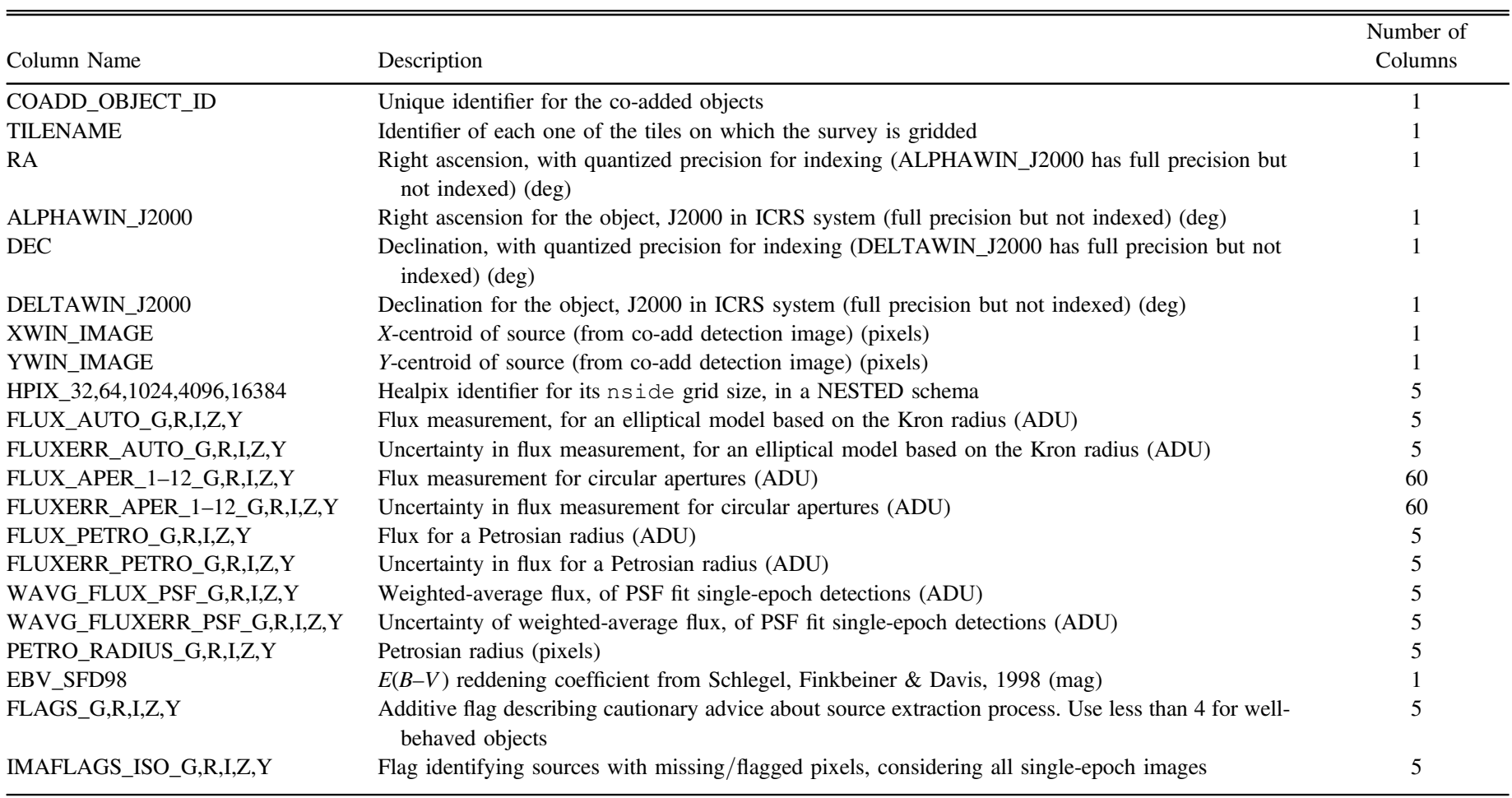


Table 10

DR1_MAGNITUDE Table Description: 399,263,026 Rows, 179 Columns

\begin{tabular}{|c|c|c|}
\hline Column Name & Description & $\begin{array}{c}\text { Number of } \\
\text { Columns }\end{array}$ \\
\hline COADD_OBJECT_ID & Unique identifier for the co-added objects & 1 \\
\hline TILENAME & Identifier of each one of the tiles on which the survey is gridded & 1 \\
\hline RA & $\begin{array}{l}\text { Right ascension, with quantized precision for indexing (ALPHAWIN_J2000 has full precision but } \\
\text { not indexed) (deg) }\end{array}$ & 1 \\
\hline ALPHAWIN_J2000 & Right ascension for the object, J2000 in ICRS system (full precision but not indexed) (deg) & 1 \\
\hline DEC & $\begin{array}{l}\text { Declination, with quantized precision for indexing (DELTAWIN_J2000 has full precision but not } \\
\text { indexed) (deg) }\end{array}$ & 1 \\
\hline DELTAWIN_J2000 & Declination for the object, J2000 in ICRS system (full precision but not indexed) (deg) & 1 \\
\hline XWIN_IMAGE & $X$-centroid from windowed measurements on co-added image (pixels) & 1 \\
\hline YWIN_IMAGE & $Y$-centroid from windowed measurements on co-added image (pixels) & 1 \\
\hline HPIX_32,64,1024,4096,16384 & Healpix identifier for its nside grid size, in a NESTED schema & 5 \\
\hline MAG_AUTO_G,R,I,Z,Y & Magnitude estimation, for an elliptical model based on the Kron radius (mag) & 5 \\
\hline MAGERR_AUTO_G,R,I,Z,Y & Uncertainty in magnitude estimation, for an elliptical model based on the Kron radius (mag) & 5 \\
\hline MAG_APER_1-12_G,R,I,Z,Y & Magnitude estimation for circular apertures (mag) & 60 \\
\hline MAGERR_APER_1-12_G,R,I,Z,Y & Uncertainty in magnitude estimation for circular apertures (mag) & 60 \\
\hline MAG_PETRO_G,R,I,Z,Y & Magnitude for a Petrosian radius (mag) & 5 \\
\hline MAGERR_PETRO_G,R,I,Z,Y & Uncertainty in magnitude for a Petrosian radius (mag) & 5 \\
\hline WAVG_MAG_PSF_G,R,I,Z,Y & Weighted-average magnitude, of PSF fit single-epoch detections (mag) & 5 \\
\hline WAVG_MAGERR_PSF_G,R,I,Z,Y & Uncertainty of weighted-average magnitude, of PSF fit single-epoch detections (mag) & 5 \\
\hline PETRO_RADIUS_G,R,I,Z,Y & Petrosian radius (pixels) & 5 \\
\hline EBV_SFD98 & $E(B-V)$ reddening coefficient from Schlegel, Finkbeiner \& Davis, $1998(\mathrm{mag})$ & 1 \\
\hline FLAḠS_G,R,I,Z,Y & $\begin{array}{l}\text { Additive flag describing cautionary advice about source extraction process. Use less than } 4 \text { for well- } \\
\text { behaved objects }\end{array}$ & 5 \\
\hline IMAFLAGS_ISO_G,R,I,Z,Y & Flag identifying sources with missing/flagged pixels, considering all single-epoch images & 5 \\
\hline
\end{tabular}

Table 11

DR1_TILE_INFO Table Description: 10,338 Rows, 46 Columns

\begin{tabular}{|c|c|c|}
\hline Column Name & Description & Number of Columns \\
\hline TILENAME & Tilename identifier & 1 \\
\hline RA_CENT & Central right ascension for tile (deg) & 1 \\
\hline DEC_CENT & Central declination for tile (deg) & 1 \\
\hline COUNT & Number of objects per tile & 1 \\
\hline RAC1 & Right ascension at corner 1 of tile (deg) & 1 \\
\hline RAC2 & Right ascension at corner 2 of tile (deg) & 1 \\
\hline RAC3 & Right ascension at corner 3 of tile (deg) & 1 \\
\hline RAC4 & Right ascension at corner 4 of tile (deg) & 1 \\
\hline RACMAX & Maximum right ascension covered in tile (deg) & 1 \\
\hline RACMIN & Minimum right ascension covered in tile (deg) & 1 \\
\hline RA_SIZE & Extent of right ascension for tile (deg) & 1 \\
\hline URAMAX & Maximum unique right ascension of objects measured from tile (deg) & 1 \\
\hline URAMIN & Minimum unique right ascension of objects measured from tile (deg) & 1 \\
\hline DECC1 & Declination at corner 1 of tile $(\mathrm{deg})$ & 1 \\
\hline DECC2 & Declination at corner 2 of tile (deg) & 1 \\
\hline DECC3 & Declination at corner 3 of tile (deg) & 1 \\
\hline DECC4 & Declination at corner 4 of tile (deg) & 1 \\
\hline DECCMAX & Maximum declination covered in tile (deg) & 1 \\
\hline DECCMIN & Minimum declination covered in tile (deg) & 1 \\
\hline DEC_SIZE & Extent of declination for tile, in average is $0.7304 \mathrm{deg}(\mathrm{deg})$ & 1 \\
\hline UDECMAX & Maximum unique declination of objects measured from tile (deg) & 1 \\
\hline UDECMIN & Minimum unique declination of objects measured from tile (deg) & 1 \\
\hline CTYPE1 & WCS projection used for axis 1 . Value: RA-TAN & 1 \\
\hline CTYPE2 & WCS projection used for axis 2 . Value: DEC-TAN & 1 \\
\hline NAXIS1 & WCS definition for number of pixels for axis 1 & 1 \\
\hline NAXIS2 & WCS definition for number of pixels for axis 2 & 1 \\
\hline CRPIX1 & WCS definition of central pixel for axis 1 . Value: 5000.5 & 1 \\
\hline CRPIX2 & WCS definition of central pixel for axis 2. Value: 5000.5 & 1 \\
\hline CRVAL1 & WCS definition of central pixel value for axis 1 & 1 \\
\hline CRVAL2 & WCS definition of central pixel value for axis 2 & 1 \\
\hline
\end{tabular}


Table 11

(Continued)

\begin{tabular}{|c|c|c|}
\hline Column Name & Description & Number of Columns \\
\hline CD1_1 & WCS definition for pixel orientation. Value: -0.0000730556 & 1 \\
\hline CD1_2 & WCS definition for pixel orientation. Value: 0 & 1 \\
\hline CD2_1 & WCS definition for pixel orientation. Value: 0 & 1 \\
\hline CD2_2 & WCS definition for pixel orientation. Value: 0.0000730556 & 1 \\
\hline CROSSRA0 & Flag tile boundary crosses $\mathrm{RA}=0 / 24 \mathrm{~h}$ boundary. Values: $\mathrm{Y}$ or $\mathrm{N}$ & 1 \\
\hline PIXELSCALE & WCS definition of pixel scale. Values: $0.263 \mathrm{arcsec} / \mathrm{pixel}[\mathrm{arcsec} / \mathrm{pixel}]$ & 1 \\
\hline TIFF_COLOR_IMAGE & Filename of the TIFF image for the tile, being tilename_r $\{$ reqnum $\}$ p $\{$ attnum $\} \_$irg.tiff & 1 \\
\hline FITS_DR1_FLUX & Filename of the served FITS being tilename_dr1_flux.fits.fz & 1 \\
\hline FITS_DR1_MAGNITUDE & Filename of the served FITS being tilename_dr1_magnitude.fits.fz & 1 \\
\hline FITS_DR1_MAIN & Filename of the served FITS being tilename_dr1_main.fits.fz & 1 \\
\hline FITS_IMAGE_DET & Filename of the served FITS being tilename_r $\{$ reqnum $\}$ p $\{$ attnum $\} \_d e t . f i t s . f z$ & 1 \\
\hline FITS_IMAGE_G,R,I,Z,Y & Filename of the served FITS, per band, being tilename_r $r\{$ reqnum $\}$ p $\{$ attnum $\}$ band.fits.fz & 5 \\
\hline
\end{tabular}

\section{ORCID iDs}

J. Annis (i) https://orcid.org/0000-0002-0609-3987

S. Bocquet (1) https://orcid.org/0000-0002-4900-805X

D. Brooks (ㄴ) https://orcid.org/0000-0002-8458-5047

D. L. Burke (16) https://orcid.org/0000-0003-1866-1950

M. Carrasco Kind (i) https://orcid.org/0000-0002-4802-3194

F. J. Castander (i) https://orcid.org/0000-0001-7316-4573

C. Conselice (ib https://orcid.org/0000-0003-1949-7638

J. DeRose (i) https://orcid.org/0000-0002-0728-0960

A. Drlica-Wagner (i) https://orcid.org/0000-0001-8251-933X

J. García-Bellido (i) https://orcid.org/0000-0002-9370-8360

D. W. Gerdes (1) https://orcid.org/0000-0001-6942-2736

K. Glazebrook (1) https://orcid.org/0000-0002-3254-9044

D. A. Goldstein (10) https://orcid.org/0000-0003-3461-8661

D. Gruen (1) https://orcid.org/0000-0003-3270-7644

R. A. Gruendl (1) https://orcid.org/0000-0002-4588-6517

S. Hamilton (1) https://orcid.org/0000-0002-6126-8487

D. Hollowood (1) https://orcid.org/0000-0002-9369-4157

K. Kuehn (i) https://orcid.org/0000-0003-0120-0808

P. Martini (i) https://orcid.org/0000-0002-4279-4182

R. G. McMahon (1) https://orcid.org/0000-0001-8447-8869

P. Melchior (10) https://orcid.org/0000-0002-8873-5065

R. Miquel (i) https://orcid.org/0000-0002-6610-4836

E. Morganson (1) https://orcid.org/0000-0001-7180-109X

J. Mould (i) https://orcid.org/0000-0003-3820-1740

B. Nord (1) https://orcid.org/0000-0001-6706-8972

P. Nugent $\odot$ ib https://orcid.org/0000-0002-3389-0586

A. Palmese (1) https://orcid.org/0000-0002-6011-0530

P. M. Ricker (1) https://orcid.org/0000-0002-5294-0630

A. K. Romer (1) https://orcid.org/0000-0002-9328-879X

A. Roodman (ib https://orcid.org/0000-0001-5326-3486

N. Shipp (i) https://orcid.org/0000-0003-2497-091X

M. Smith (i) https://orcid.org/0000-0002-3321-1432

M. Soares-Santos (i) https://orcid.org/0000-0001-6082-8529

F. Sobreira (1) https://orcid.org/0000-0002-7822-0658

M. Sullivan (1) https://orcid.org/0000-0001-9053-4820

G. Tarle (i) https://orcid.org/0000-0003-1704-0781

A. K. Vivas (1) https://orcid.org/0000-0003-4341-6172

A. R. Walker (i) https://orcid.org/0000-0002-7123-8943

R. H. Wechsler (1) https://orcid.org/0000-0003-2229-011X

B. Yanny (1) https://orcid.org/0000-0002-9541-2678

S. Juneau (1) https://orcid.org/0000-0002-0000-2394

D. Nidever (1) https://orcid.org/0000-0002-1793-3689

K. Olsen (i) https://orcid.org/0000-0002-7134-8296

\section{References}

Abazajian, K. N., Adelman-McCarthy, J. K., Agüeros, M. A., et al. 2009 , ApJS, 182, 543

Aihara, H., Arimoto, N., Armstrong, R., et al. 2018a, PASJ, 70, 4

Aihara, H., Armstrong, R., Bickerton, S., et al. 2018b, PASJ, 70, 8

Astropy Collaboration, Robitaille, T. P., Tollerud, E. J., et al. 2013, A\&A, 558, A33

Berk, A., Anderson, G. P., Bernstein, L. S., et al. 1999, Proc. SPIE, 3756, 348 Bernstein, G. M., Abbott, T. M. C., Desai, S., et al. 2017a, PASP, 129, 114502 Bernstein, G. M., Armstrong, R., Plazas, A. A., et al. 2017b, PASP, 129, 074503 Bertin, E. 2006, in ASP Conf. Ser. 351, Astronomical Data Analysis Software and Systems XV, ed. C. Gabriel et al. (San Francisco, CA: ASP), 112

Bertin, E. 2010, SWarp: Resampling and Co-adding FITS Images Together, Astrophysics Source Code Library, ascl:1010.068

Bertin, E. 2011, in ASP Conf. Ser. 442, Astronomical Data Analysis Software and Systems XX, ed. I. N. Evans et al. (San Francisco, CA: ASP), 435

Bertin, E. 2012, in ASP Conf. Ser. 461, Astronomical Data Analysis Software and Systems XXI, ed. P. Ballester, D. Egret, \& N. P. F. Lorente (San Francisco, CA: ASP), 263

Bertin, E., \& Arnouts, S. 1996, A\&As, 117, 393

Bertin, E., Mellier, Y., Radovich, M., et al. 2002, in ASP Conf. Ser. 281, Astronomical Data Analysis Software and Systems XI, ed. D. A. Bohlender, D. Durand, \& T. H. Handley (San Francisco, CA: ASP), 228

Bertin, E., Pillay, R., \& Marmo, C. 2015, A\&C, 10, 43

Bohlin, R. C., Gordon, K. D., \& Tremblay, P.-E. 2014, PASP, 126, 711

Burke, D., Rykoff, E. S., Allam, S., et al. 2017, arXiv:1706.01542

Burke, D. L., Rykoff, E. S., Allam, S., et al. 2018, AJ, 155, 41

Calabretta, M. R., \& Greisen, E. W. 2002, A\&A, 395, 1077

Carlstrom, J. E., Ade, P. A. R., Aird, K. A., et al. 2011, PASP, 123, 568

Carrasco Kind, M., Drlica-Wagner, A., Koziol, A. M. G., \& Petravick, D. 2018, arXiv:1810.02721

Chang, C., Busha, M. T., Wechsler, R. H., et al. 2015, ApJ, 801, 73

Dark Energy Survey Collaboration, Abbott, T., Abdalla, F. B., et al. 2016, MNRAS, 460, 1270

de Jong, J. T. A., Verdoes Kleijn, G. A., Kuijken, K. H., \& Valentijn, E. A. 2013, ExA, 35, 25

DES Collaboration 2005, arXiv:astro-ph/0510346

DES Collaboration 2017, arXiv:1708.01530

Desai, S., Armstrong, R., Mohr, J. J., et al. 2012, ApJ, 757, 83

Diehl, H. T., Neilsen, E., Gruendl, R., et al. 2014, Proc. SPIE, 9149, 91490V

Diehl, H. T., Neilsen, E., Gruendl, R., et al. 2016, Proc. SPIE, 9910, 99101D

Drlica-Wagner, A., Sevilla-Noarbe, I., Rykoff, E. S., et al. 2018, ApJS, 235, 33

Erben, T., Hildebrandt, H., Miller, L., et al. 2013, MNRAS, 433, 2545

Fadely, R., Hogg, D. W., \& Willman, B. 2012, ApJ, 760, 15

Fitzpatrick, E. L. 1999, PASP, 111, 63

Fitzpatrick, M. 2010, in ASP Conf. Ser. 434, Astronomical Data Analysis Software and Systems XIX, ed. Y. Mizumoto, K.-I. Morita, \& M. Ohishi (San Francisco, CA: ASP), 260

Fitzpatrick, M. J., Graham, M. J., Mighell, K. J., et al. 2016, Proc. SPIE, 9913, 99130L

Flaugher, B., Diehl, H. T., Honscheid, K., et al. 2015, AJ, 150, 150

Gaia Collaboration, Brown, A. G. A., Vallenari, A., et al. 2016, A\&A, 595, A2

Górski, K. M., Hivon, E., Banday, A. J., et al. 2005, ApJ, 622, 759 
Greisen, E. W., \& Calabretta, M. R. 2002, A\&A, 395, 1061

Gruen, D., Bernstein, G. M., Jarvis, M., et al. 2015, JInst, 10, C05032

Hamilton, A. J. S., \& Tegmark, M. 2004, MNRAS, 349, 115

Harris, W. E. 1996, AJ, 112, 1487

Honscheid, K., DePoy, D. L. \& for the DES Collaboration 2008, arXiv:0810.3600

Honscheid, K., Elliott, A., Annis, J., et al. 2012, Proc. SPIE, 8451, 845112

Hunter, J. D. 2007, CSE, 9, 90

Ivezic, Z., Tyson, J. A., Abel, B., et al. 2008, arXiv:0805.2366

Jones, E., Oliphant, T., Peterson, P., et al. 2001, SciPy: Open Source Scientific Tools for Python, http://www.scipy.org/

Jordi, C., Gebran, M., Carrasco, J. M., et al. 2010, A\&A, 523, A48

Kaiser, N., Burgett, W., Chambers, K., et al. 2010, Proc. SPIE, 7733, $77330 \mathrm{E}$

Kessler, R., Marriner, J., Childress, M., et al. 2015, AJ, 150, 172

Kim, E. J., \& Brunner, R. J. 2017, MNRAS, 464, 4463

Kim, E. J., Brunner, R. J., \& Carrasco Kind, M. 2015, MNRAS, 453, 507

Koposov, S., \& Bartunov, O. 2006, ASP Conf. Ser. 351, Astronomical Data Analysis Software and Systems XV, ed. C. Gabriel et al. (San Francisco, CA: ASP), 735

Małek, K., Solarz, A., Pollo, A., et al. 2013, A\&A, 557, A16

Marshall, J. L., Rheault, J.-P., DePoy, D. L., et al. 2013, arXiv:1302.5720

Mohr, J. J., Armstrong, R., Bertin, E., et al. 2012, Proc. SPIE, 8451, 84510D

Morganson, E., Gruendl, R. A., Menanteau, F., et al. 2018, PASP, 130, 074501
Neilsen, E., \& Annis, J. 2014, in ASP Conf. Ser. 485, Astronomical Data Analysis Software and Systems XXIII, ed. N. Manset \& P. Forshay (San Francisco, CA: ASP), 77

Oke, J. B. 1974, ApJS, 27, 21

Pogson, N. 1856, MNRAS, 17, 12

Reed, S. L., McMahon, R. G., Banerji, M., et al. 2015, MNRAS, 454, 3952

Rykoff, E. S., Rozo, E., \& Keisler, R. 2015, arXiv:1509.00870

Schlafly, E. F., \& Finkbeiner, D. P. 2011, ApJ, 737, 103

Schlegel, D. J., Finkbeiner, D. P., \& Davis, M. 1998, ApJ, 500, 525

Sevilla, I., Armstrong, R., Bertin, E., et al. 2011, arXiv:1109.6741

Sevilla-Noarbe, I., Hoyle, B., Marchã, M. J., et al. 2018, arXiv:1805.02427

Sheldon, E. S. 2014, MNRAS, 444, L25

Skrutskie, M. F., Cutri, R. M., Stiening, R., et al. 2006, AJ, 131, 1163

Soumagnac, M. T., Abdalla, F. B., Lahav, O., et al. 2015, MNRAS, 450, 666

Swanson, M. E. C., Tegmark, M., Hamilton, A. J. S., \& Hill, J. C. 2008, MNRAS, 387, 1391

Taylor, M. B. 2005, in ASP Conf. Ser. 347, Astronomical Data Analysis Software and Systems XIV, ed. P. Shopbell, M. Britton, \& R. Ebert (San Francisco, CA: ASP), 29

The HDF Group 1997, NNNN, Hierarchical Data Format, version 5, http:// www.hdfgroup.org/HDF5/

Tyson, J. A. 2010, Proc. SPIE, 7733, 773303

Van Der Walt, S., Colbert, S. C., \& Varoquaux, G. 2011, CSE, 13, 22

Wells, D. C., Greisen, E. W., \& Harten, R. H. 1981, A\&AS, 44, 363

York, D. G., Adelman, J., Anderson, J. E., Jr., et al. 2000, AJ, 120, 1579 